\title{
Effect of Exocyclic Substituents and $\pi$-System Length on the Electronic Structure of Chichibabin Diradicaloids
}

Sílvia Escayola, ${ }^{\ddagger}$ Marc Callís, $₫$ Albert Poater, $\$ * *$ and Miquel Solà, $\$, *$

\#nstitut de Química Computacional i Catàlisi and Departament de Química, Universitat de Girona, C/ Maria Aurèlia Capmany, 69, 17003 Girona, Catalonia, Spain.

Corresponding authors:

*E-mail: albert.poater@udg.edu

*E-mail: $\underline{\text { miquel.sola@udg.edu }}$

\section{Table of Contents}

I. Selection of the Computational Approach

II. Analysis of the different conformations

III. Structural parameters, energy, $<\hat{S}^{2}>$, diradical character $(y)$, Mulliken populations and aromaticity results

IV. References 


\section{Selection of the Computational Approach}

In order to determine the most appropriate density functional theory (DFT) method to study the geometries and singlet-triplet energy gap $\left(\Delta \mathrm{E}_{\mathrm{S}-\mathrm{T}}\right)$ of $p$-xylylene-like hydrocarbons (HCs), we have compared the performance of distinct functionals with the $\operatorname{CCSD}(\mathrm{T})$ results. Eleven DFT functionals (BLYP, BP86, PBE, OPBE, M06-L, TPSS, MN15, MN15-L, B3LYP, CAM-B3LYP and $\omega-\mathrm{B} 97 \mathrm{xD}$ ) including generalized gradient approximation (GGA), meta-GGA, hybrid GGA and long-range corrected functionals have been evaluated. For the B3LYP functional we also tested to include the GD3BJ ${ }^{[1]}$ empirical dispersion. The geometry optimizations were performed considering the three electronic states: singlet closed-shell (CS), singlet open-shell (OSS) and triplet (T) of compounds I and II (Figure S1). In all the cases, restricted and unrestricted (R and U followed by the method) DFT/cc-pVTZ calculations were carried out to optimize the CS and OSS states, respectively. Moreover, in the OS singlet case we request the HOMO-LUMO mixing to break the $\alpha-\beta$ and spatial symmetries (guess $=$ mix Gaussian keyword). However, in most of the cases we obtained the same CS solution for both calculations, the restricted and the unrestricted one. As definitive proof, we employ the broken-symmetry (BS) ${ }^{[2]}$ UDFT approach with explicit definition of charge and multiplicity by fragments, trying force the diradicaloid situation, but we obtain very small differences (in the range or the DFT error) with respect the CS results (table S1).

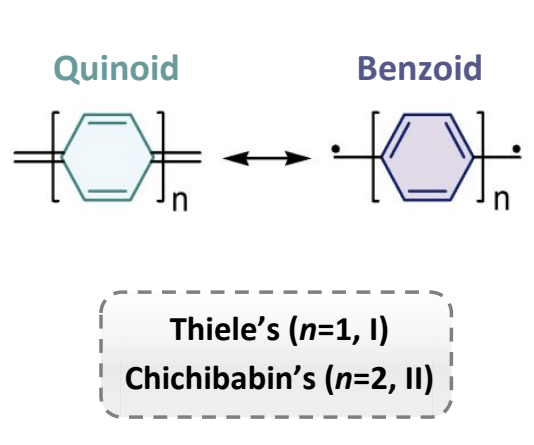

Figure S1. Quinoid and benzoid resonance forms of Thiele's $(n=1)$ and Chichibabin's $(n=2)$ HCs.

\begin{tabular}{ccc:cc}
\hline \multirow{2}{*}{ Functional } & \multicolumn{2}{c}{ BS $\Delta \mathbf{E}$ (cs-oss) (eV) } & \multicolumn{2}{|c}{$\Delta \mathbf{E}$ (cs-oss) $\mathbf{( e V )}$} \\
& I & II & I & II \\
\hline BP86 & 0.00 & 0.00 & 0.00 & 0.00 \\
B3LYP & 0.00 & 0.00 & 0.00 & -0.01 \\
B3LYP-GD3BJ & 0.00 & 0.00 & 0.00 & 0.00 \\
CAM-B3LYP & 0.00 & -0.13 & 0.00 & -0.13 \\
wB97xD & 0.00 & -0.14 & 0.00 & 0.00 \\
BLYP & 0.00 & 0.00 & 0.00 & 0.00 \\
PBE & 0.00 & 0.00 & 0.00 & 0.00 \\
OPBE & 0.00 & 0.00 & 0.00 & 0.00 \\
TPSS & 0.00 & 0.00 & 0.00 & 0.00 \\
M06-L & 0.00 & 0.00 & 0.00 & 0.00 \\
MN15 & 0.00 & 0.00 & 0.00 & -0.01 \\
MN15-L & 0.00 & 0.00 & 0.00 & 0.00 \\
\hline
\end{tabular}

Table S1. $\Delta \mathrm{E}$ (cs-os) values for the different tested functionals. $\Delta \mathrm{E}$ computed with the $\mathrm{BS}$ results (right), non-BS (left).

We have optimized the test systems at the QCISD/cc-pVDZ level of theory, and we have used these optimized geometries for comparing structural parameters (i.e. bond distances and angles) with those obtained with DFT (Figure S2 and Table S2). For the evaluation of the $\Delta \mathrm{E}_{\mathrm{S}-\mathrm{T}}$, we took the energies form the the CCSD(T)/cc-pVDZ single point calculations of the optimized QCISD geometries as reference values (Table S3). All calculations were performed with the Gaussian 16 revision A.03 program. ${ }^{[3]}$ 

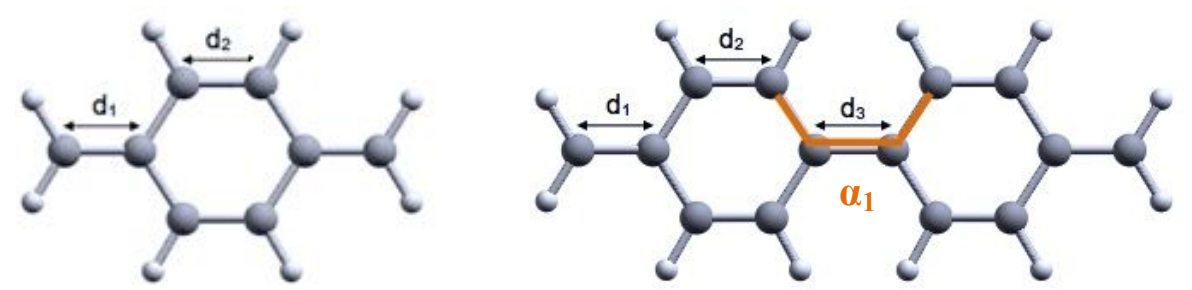

Figure S2. Selected bond lengths $d_{1}, d_{2}$ (and $d_{3}$ and dihedral angle $\left(\alpha_{1}\right)$ ) for system I (and II).

Table S2. Values of the bond distances $\mathrm{d}_{1}, \mathrm{~d}_{2}$ (and $\mathrm{d}_{3}$ and dihedral angle $\left(\alpha_{1}\right)$ ) for system I (and II) calculated using different DFT functionals and QCISD/cc-pVDZ (reference results highlighted in blue). The represented distances and angles have $\AA$ and degrees units, respectively.

\begin{tabular}{|c|c|c|c|c|c|c|c|c|c|c|c|c|c|c|c|}
\hline & & & & & & & $d_{1}(\AA)$ & & & & & & & & \\
\hline Structure & state & QCISD & BP86 & B3LYP & B3LYP-GD3BJ & BS-B3LYP & CAM-B3LYP & wB97xD & BLYP & PBE & TPSS & M06-L & MN15 & MN15-L & OPBE \\
\hline \multirow{3}{*}{ I } & $\mathrm{CS}$ & 1.360 & 1.361 & 1.348 & 1.347 & - & 1.337 & 1.339 & 1.362 & 1.360 & 1.358 & 1.350 & 1.343 & 1.361 & 1.360 \\
\hline & OSS & 1.360 & 1.361 & 1.348 & 1.347 & 1.348 & 1.337 & 1.339 & 1.362 & 1.360 & 1.358 & 1.350 & 1.343 & 1.361 & 1.360 \\
\hline & $\mathrm{T}$ & 1.456 & 1.435 & 1.434 & 1.432 & - & 1.434 & 1.435 & 1.437 & 1.433 & 1.437 & 1.428 & 1.433 & 1.443 & 1.434 \\
\hline & $\mathrm{CS}$ & 1.363 & 1.369 & 1.354 & 1.140 & - & 1.340 & 1.341 & 1.370 & 1.368 & 1.366 & 1.357 & 1.347 & 1.368 & 1.367 \\
\hline \multirow[t]{2}{*}{ II } & OSS & 1.363 & 1.369 & 1.360 & 1.358 & 1.354 & 1.371 & 1.342 & 1.369 & 1.368 & 1.366 & 1.357 & 1.347 & 1.368 & 1.367 \\
\hline & $\mathrm{T}$ & 1.417 & 1.406 & 1.402 & 1.401 & - & 1.400 & 1.402 & 1.407 & 1.405 & 1.408 & 1.399 & 1.402 & 1.414 & 1.406 \\
\hline & \multicolumn{15}{|c|}{$d_{2}(\AA)$} \\
\hline Structure & state & QCISD & BP86 & B3LYP & B3LYP-GD3BJ & BS-B3LYP & CAM-B3LYP & wB97xD & BLYP & PBE & TPSS & M06-L & MN15 & MN15-L & OPBE \\
\hline \multirow{4}{*}{ I } & $\mathrm{CS}$ & 1.358 & 1.355 & 1.343 & 1.342 & - & 1.333 & 1.335 & 1.355 & 1.354 & 1.352 & 1.344 & 1.339 & 1.355 & 1.352 \\
\hline & OSS & 1.358 & 1.355 & 1.343 & 1.342 & 1.343 & 1.333 & 1.335 & 1.355 & 1.354 & 1.352 & 1.344 & 1.339 & 1.355 & 1.352 \\
\hline & $\mathrm{T}$ & 1.402 & 1.391 & 1.385 & 1.384 & - & 1.380 & 1.382 & 1.393 & 1.390 & 1.389 & 1.381 & 1.384 & 1.393 & 1.387 \\
\hline & $\mathrm{CS}$ & 1.362 & 1.363 & 1.350 & 1.100 & - & 1.339 & 1.340 & 1.364 & 1.362 & 1.360 & 1.352 & 1.345 & 1.363 & 1.360 \\
\hline \multirow[t]{2}{*}{ II } & OSS & 1.362 & 1.363 & 1.354 & 1.353 & 1.350 & 1.358 & 1.340 & 1.364 & 1.362 & 1.360 & 1.352 & 1.345 & 1.363 & 1.360 \\
\hline & $\mathrm{T}$ & 1.393 & 1.385 & 1.378 & 1.377 & - & 1.374 & 1.376 & 1.386 & 1.384 & 1.383 & 1.375 & 1.377 & 1.388 & 1.382 \\
\hline & \multicolumn{15}{|c|}{$\mathrm{d}_{3}(\AA)$} \\
\hline Structure & state & QCISD & BP86 & B3LYP & B3LYP-GD3BJ & BS-B3LYP & CAM-B3LYP & wB97xD & BLYP & PBE & TPSS & M06-L & MN15 & MN15-L & OPBE \\
\hline \multirow{3}{*}{ II } & CS & 1.394 & 1.419 & 1.400 & 1.100 & - & 1.378 & 1.377 & 1.421 & 1.418 & 1.415 & 1.406 & 1.386 & 1.418 & 1.417 \\
\hline & OSS & 1.394 & 1.419 & 1.411 & 1.408 & 1.400 & 1.435 & 1.377 & 1.421 & 1.418 & 1.415 & 1.406 & 1.386 & 1.418 & 1.418 \\
\hline & $\mathrm{T}$ & 1.494 & 1.478 & 1.478 & 1.475 & - & 1.479 & 1.480 & 1.483 & 1.476 & 1.478 & 1.468 & 1.477 & 1.482 & 1.476 \\
\hline & \multicolumn{15}{|c|}{$\alpha_{1}$ (degrees) } \\
\hline Structure & state & QCISD & BP86 & B3LYP & B3LYP-GD3BJ & BS-B3LYP & CAM-B3LYP & wB97xD & BLYP & PBE & TPSS & M06-L & MN15 & MN15-L & OPBE \\
\hline \multirow{3}{*}{ II } & CS & 0.00 & 0.00 & 0.00 & 0.00 & - & 0.00 & 0.00 & 0.00 & 0.00 & 0.00 & 0.00 & 0.00 & 0.00 & -3.75 \\
\hline & OSS & 0.00 & 0.00 & 0.04 & 0.04 & -0.03 & -13.56 & 0.01 & 0.00 & 0.00 & 0.00 & 0.00 & 0.00 & 0.00 & -3.78 \\
\hline & $\mathrm{T}$ & -46.43 & -35.28 & -38.49 & -37.81 & - & -41.65 & -42.60 & -36.34 & -34.96 & -34.19 & -30.57 & -37.72 & -35.05 & -41.87 \\
\hline
\end{tabular}


Table S3. Relative energies a (with respect the CS singlet energy of each method in eV) of the closed-shell singlet and triplet states of compounds I and II obtained with different methods together with cc-pVTZ basis set. The relative error (with respect the CCSD(T)/cc-pVDZ//QCISD/cc-pVDZ $\Delta \mathrm{E}_{\mathrm{CS}-\mathrm{T}}$ in eV) of $\Delta \mathrm{E}_{\mathrm{CS}-\mathrm{T}}$ is represented in parentheses.

\begin{tabular}{|c|c|c|c|c|}
\hline \multirow{2}{*}{ method } & \multirow{2}{*}{ Compound } & \multicolumn{3}{|c|}{$\Delta E(e V)$} \\
\hline & & CS & & $\mathbf{T}$ \\
\hline \multirow{2}{*}{$\operatorname{CCSD}(\mathrm{T}) / / \mathrm{QCISD}$} & $\mathbf{I}$ & 0.00 & 1.53 & $(0.00 \%)$ \\
\hline & II & 0.00 & 0.55 & $(0.00 \%)$ \\
\hline \multirow{2}{*}{$\operatorname{CASSCF}(6,6) / / \mathrm{QCISD}$} & I & 0.00 & 1.84 & $(20.11 \%)$ \\
\hline & II & 0.00 & 1.07 & $(93.13 \%)$ \\
\hline \multirow{2}{*}{$\mathrm{CCSD}(\mathrm{T}) / / \mathrm{MP} 2$} & $\mathbf{I}$ & 0.00 & 1.53 & $(0.07 \%)$ \\
\hline & II & 0.00 & 0.92 & $(67.15 \%)$ \\
\hline \multirow{2}{*}{ BP86 } & $\mathbf{I}$ & 0.00 & 1.46 & $(4.62 \%)$ \\
\hline & II & 0.00 & 0.54 & $(1.55 \%)$ \\
\hline \multirow{2}{*}{ B3LYP } & $\mathbf{I}$ & 0.00 & 1.42 & $(7.38 \%)$ \\
\hline & II & 0.00 & 0.36 & $(34.59 \%)$ \\
\hline \multirow{2}{*}{ B3LYP-GD3BJ } & $\mathbf{I}$ & 0.00 & 1.42 & $(7.10 \%)$ \\
\hline & II & 0.00 & 0.37 & $(33.45 \%)$ \\
\hline \multirow{2}{*}{ CAM-B3LYP } & $\mathbf{I}$ & 0.00 & 1.37 & $(10.74 \%)$ \\
\hline & II & 0.00 & 0.12 & $(77.87 \%)$ \\
\hline \multirow{2}{*}{ wB97xD } & $\mathbf{I}$ & 0.00 & 1.37 & $(10.34 \%)$ \\
\hline & II & 0.00 & 0.08 & $(85.81 \%)$ \\
\hline \multirow{2}{*}{ BLYP } & $\mathbf{I}$ & 0.00 & 1.50 & $(1.93 \%)$ \\
\hline & II & 0.00 & 0.58 & $(4.06 \%)$ \\
\hline \multirow{2}{*}{ PBE } & I & 0.00 & 1.46 & $(4.95 \%)$ \\
\hline & II & 0.00 & 0.54 & $(2.63 \%)$ \\
\hline \multirow{2}{*}{ OPBE } & I & 0.00 & 1.39 & $(9.50 \%)$ \\
\hline & II & 0.00 & 0.44 & $(20.79 \%)$ \\
\hline \multirow{2}{*}{ TPSS } & $\mathbf{I}$ & 0.00 & 1.36 & $(10.97 \%)$ \\
\hline & II & 0.00 & 0.45 & $(18.11 \%)$ \\
\hline \multirow{2}{*}{ M06-L } & I & 0.00 & 1.40 & $(8.81 \%)$ \\
\hline & II & 0.00 & 0.47 & $(15.71 \%)$ \\
\hline \multirow{2}{*}{ MN15 } & I & 0.00 & 1.51 & $(1.62 \%)$ \\
\hline & II & 0.00 & 0.33 & $(40.11 \%)$ \\
\hline \multirow{2}{*}{ MN15-L } & I & 0.00 & 1.47 & $(4.10 \%)$ \\
\hline & II & 0.00 & 0.48 & $(12.56 \%)$ \\
\hline
\end{tabular}

${ }^{a}$ Since the OSS calculations gives the CS solution in most of the cases we just compare CS and T results.

Among all tested methods, the ones that give closer results to the reference QCISD method are the pure functionals, presenting small differences between themselves. Regarding the energy values, BP86, BLYP, PBE, OPBE, M06-L and MN15-L give the best results, with errors below $0.13 \mathrm{eV}$. Recently, C. Herrmann and coworkers published a study comparing the capability of BP86, TPSS, TPSSh and B3LYP, to reproduce experimental structural values of diradical compounds, which states that TPSSh (hybrid functional with 10\% HF exchange) shows the best results for two Chichibabin system derivatives. However, they state that none of the functionals is suitable for correctly describing relative bond lengths, and none of them is recommended for 
predictive studies. ${ }^{[4]}$ Prior studies from 2010 and 2011 M. Swart and coworkers ${ }^{[5,6]}$ determine that OPBE pure functional works very well for predicting spin states. Moreover, the OPBE gives good results for predicting magnetic shielding, which will be important for the calculations of the NICS values. ${ }^{[7]}$ Therefore, based on our own results and the results found in the literature we will use OPBE in our main study.

Finally, to see the effect of the long range corrections (LC) on the systems with large chain lengths we perform calculations at DFT level including LC for systems with $n$ from 1 to 5 (table S4).

Table S4. Relative energies (with respect the CS singlet energy of each method in $\mathrm{eV}$ ) and $<\mathrm{S}^{2}>$ values of the closed-shell singlet open-shell singlet and triplet states of Chichibabin compounds (with $\mathrm{H}$ as exocyclic substituent) with $n$ from 1 to 5 obtained with different methods together with cc-pVTZ basis set.

\begin{tabular}{|c|c|c|c|c|c|}
\hline \multicolumn{6}{|c|}{ OPBE/cc-pvtz } \\
\hline \multirow{3}{*}{ Structure } & \multicolumn{3}{|c|}{$\Delta \mathbf{E}$} & \multicolumn{2}{|c|}{$<\mathrm{S} * * 2>$} \\
\hline & \multicolumn{5}{|c|}{ Electronic state } \\
\hline & $\mathrm{CS}$ & OSS & $\mathrm{T}$ & OSS & $\mathrm{T}$ \\
\hline H_1 & 0.00 & 0.00 & 1.39 & 0.00 & 2.01 \\
\hline H_2 & 0.00 & 0.00 & 0.44 & 0.00 & 2.03 \\
\hline H_3 & 0.00 & -0.09 & -0.02 & 0.87 & 2.03 \\
\hline H_4 & 0.00 & -0.31 & -0.30 & 1.02 & 2.03 \\
\hline H_5 & 0.00 & -0.43 & -0.43 & 1.03 & 2.03 \\
\hline \multicolumn{6}{|c|}{ OPBE/cc-pvtz//LC-OPBE/cc-pvtz } \\
\hline \multirow{3}{*}{ Structure } & & $\Delta \mathbf{E}$ & & & \\
\hline & \multicolumn{5}{|c|}{ Electronic state } \\
\hline & CS & OSS & $\mathrm{T}$ & OSS & $\mathrm{T}$ \\
\hline H_1 & 0.00 & -0.04 & 1.21 & 0.35 & 2.01 \\
\hline H_2 & 0.00 & -0.58 & -0.33 & 1.15 & 2.23 \\
\hline H_3 & 0.00 & -1.58 & -1.53 & 1.41 & 2.28 \\
\hline H_4 & 0.00 & -2.67 & -2.66 & 1.36 & 2.32 \\
\hline H_5 & 0.00 & -3.38 & -3.37 & 1.35 & 2.33 \\
\hline \multicolumn{6}{|c|}{ LC-OPBE/cc-pvtz } \\
\hline \multirow{3}{*}{ Structure } & & $\Delta \mathbf{E}$ & & & \\
\hline & \multicolumn{5}{|c|}{ Electronic state } \\
\hline & $\mathrm{CS}$ & OSS & $\mathrm{T}$ & OSS & $\mathrm{T}$ \\
\hline H_1 & 0.00 & 0.00 & 1.25 & 0.00 & 2.01 \\
\hline H_2 & 0.00 & -0.40 & -0.16 & 1.21 & 2.19 \\
\hline H_3 & 0.00 & -1.20 & -1.15 & 1.31 & 2.24 \\
\hline H_4 & 0.00 & -2.07 & -2.06 & 1.28 & 2.26 \\
\hline H_5 & 0.00 & -2.94 & -2.93 & 1.28 & 2.27 \\
\hline \multicolumn{6}{|c|}{ wB97xD/cc-pvtz } \\
\hline \multirow{3}{*}{ Structure } & & $\Delta \mathbf{E}$ & & & \\
\hline & \multicolumn{5}{|c|}{ Electronic state } \\
\hline & $\mathrm{CS}$ & OSS & $\mathrm{T}$ & OSS & $\mathrm{T}$ \\
\hline H_1 & 0.00 & 0.00 & 1.37 & 0.00 & 2.01 \\
\hline H_2 & 0.00 & -0.14 & 0.08 & 0.94 & 2.08 \\
\hline H_3 & 0.00 & -0.84 & -0.81 & 1.11 & 2.10 \\
\hline H_4 & 0.00 & -1.62 & -1.61 & 1.11 & 2.11 \\
\hline H_5 & 0.00 & -2.35 & -2.35 & 1.11 & 2.11 \\
\hline
\end{tabular}




\section{Analysis of the different conformations}

A part from the selection of the method, we also perform a study of the possible conformational isomers of the Chichibabin compounds depending on the dihedral angles of the structure.

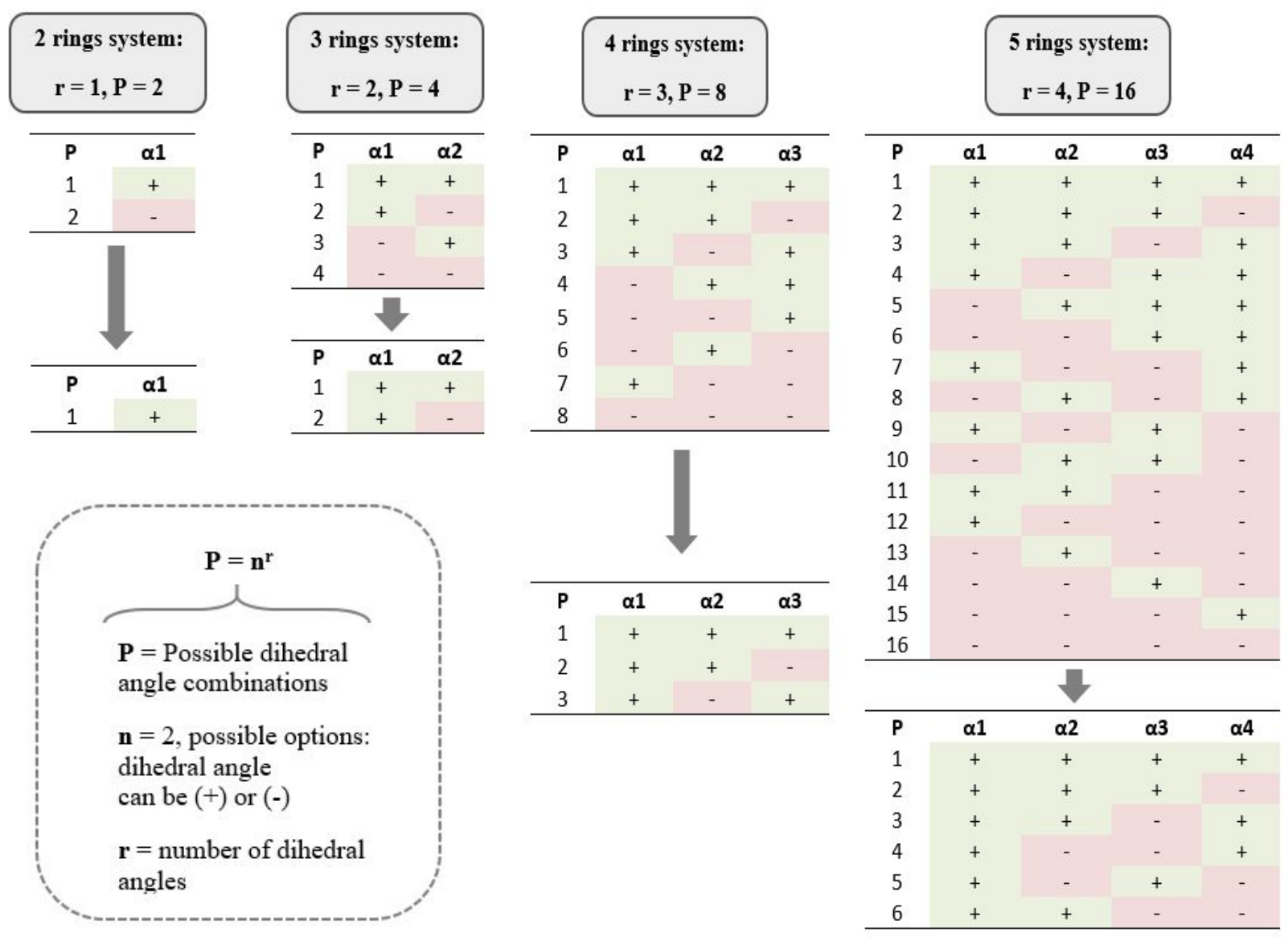

Figure S3. Different dihedral angle $(\alpha)$ signs combinations that we can have for each system. Tables below the arrows summarize the non-equivalent conformations in each case, which corresponds to the computed ones.

Table S5. OPBE/cc-pVTZ relative energies (to most stable conformer) in milielectronvolts and $\alpha$ values $\left({ }^{\circ}\right)$ of the tested conformers.

\begin{tabular}{ccccccc}
\hline no. of rings & $\mathbf{P}$ & E Rel. & $\boldsymbol{\alpha}_{\mathbf{1}}$ & $\boldsymbol{\alpha}_{\mathbf{2}}$ & $\boldsymbol{\alpha}_{\mathbf{3}}$ & $\boldsymbol{\alpha}_{\mathbf{4}}$ \\
$\mathbf{2}$ & 1 & 0.00 & -41.9 & & & \\
$\mathbf{3}$ & 1 & 3.47 & 36.9 & 36.9 & & \\
& 2 & 0.00 & 36.6 & -36.6 & & \\
$\mathbf{4}$ & 1 & 3.04 & 35.9 & 40.1 & 35.9 & \\
& 2 & 1.30 & 35.7 & 39.6 & -35.3 & \\
& 3 & 0.00 & 35.2 & -39.0 & 35.2 & \\
& 1 & 2.60 & 35.5 & 40.6 & 40.6 & 35.5 \\
$\mathbf{5}$ & 2 & 1.73 & 35.6 & 40.3 & 39.7 & -35.0 \\
& 3 & 0.43 & 35.5 & 40.0 & -39.3 & 35.1 \\
& 4 & 0.87 & 34.9 & -40.0 & -40.0 & 34.9 \\
& 5 & 0.00 & 35.0 & -39.3 & 39.4 & -35.0 \\
& 6 & 1.73 & 35.5 & 39.8 & -39.8 & -35.5 \\
\hline
\end{tabular}




\section{Structural parameters, energy, $<\hat{S}^{2}>$, Mulliken populations and aromaticity results}

In order to present the results in a clearer way we will use the alternative nomenclature from table S4 to name the different compounds in the following sections.

Table S6. Different studied Chichibabin systems nomenclature, depending on the $\mathrm{R}$ and $n$.

\begin{tabular}{|c|c|c|c|c|c|c|c|}
\hline system & $\begin{array}{l}\text { Substituent } \\
\text { (R) }\end{array}$ & $n$ & $\begin{array}{c}\text { Alternative } \\
\text { nomenclature }\end{array}$ & system & $\begin{array}{l}\text { Substituent } \\
\text { (R) }\end{array}$ & $n$ & $\begin{array}{c}\text { Alternative } \\
\text { nomenclature }\end{array}$ \\
\hline 1 & $x^{-2}$ & $\begin{array}{l}1 \\
2 \\
3 \\
4 \\
5\end{array}$ & $\begin{array}{l}\mathrm{h} \_1 \\
\mathrm{~h} \_2 \\
\mathrm{~h} \_3 \\
\mathrm{~h} \_4 \\
\mathrm{~h}-5\end{array}$ & 7 & & $\begin{array}{l}1 \\
2 \\
3 \\
4 \\
5\end{array}$ & $\begin{array}{l}\text { ph_1 } \\
\text { ph_2 } \\
\text { ph_3 } \\
\text { ph_4 } \\
\text { ph_5 }\end{array}$ \\
\hline 2 & & $\begin{array}{l}1 \\
2 \\
3 \\
4 \\
5\end{array}$ & $\begin{array}{l}\text { oh_1 } \\
\text { oh_2 } \\
\text { oh_3 } \\
\text { oh_4 } \\
\text { oh_5 }\end{array}$ & 8 & & $\begin{array}{l}1 \\
2 \\
3 \\
4 \\
5\end{array}$ & $\begin{array}{l}\text { fph_1 } \\
\text { fph_2 } \\
\text { fph_3 } \\
\text { fph_4 } \\
\text { fph_5 }\end{array}$ \\
\hline 3 & & $\begin{array}{l}1 \\
2 \\
3 \\
4 \\
5\end{array}$ & $\begin{array}{l}\mathrm{nh}{ }^{-} 1 \\
\mathrm{nh} 2 \\
\mathrm{nh}-3 \\
\mathrm{nh}{ }^{-4} \\
\mathrm{nh}-5\end{array}$ & 9 & & $\begin{array}{l}1 \\
2 \\
3 \\
4 \\
5\end{array}$ & $\begin{array}{l}\text { noph_1 } \\
\text { noph_2 } \\
\text { noph_3 } \\
\text { noph_4 } \\
\text { noph-5 }\end{array}$ \\
\hline 4 & & $\begin{array}{l}1 \\
2 \\
3 \\
4 \\
5\end{array}$ & $\begin{array}{l}\text { coh_1 } \\
\text { coh_2 } \\
\text { coh_3 } \\
\text { coh_4 } \\
\text { coh-5 }\end{array}$ & 10 & & $\begin{array}{l}1 \\
2 \\
3 \\
4 \\
5\end{array}$ & $\begin{array}{l}\text { p_fph_1 } \\
\text { p_fph_2 } \\
\text { p_fph_3 } \\
\text { p_fph_4 } \\
\text { p_fph_5 }\end{array}$ \\
\hline 5 & $\mathrm{~N} \equiv \mathrm{C}-\mathrm{O}$ & $\begin{array}{l}1 \\
2 \\
3 \\
4 \\
5\end{array}$ & $\begin{array}{l}\mathrm{cn} \_1 \\
\mathrm{cn} \_2 \\
\mathrm{cn} \_3 \\
\mathrm{cn} \_4 \\
\mathrm{cn} 55\end{array}$ & 11 & $\mathrm{O}_{2}^{\mathrm{O}_{2} \mathrm{~N}}$ & $\begin{array}{l}1 \\
2 \\
3 \\
4 \\
5\end{array}$ & $\begin{array}{l}\text { p_noph_1 } \\
\text { p_noph_2 } \\
\text { p_noph_3 } \\
\text { p_noph_4 } \\
\text { p_noph_5 }\end{array}$ \\
\hline 6 & & $\begin{array}{l}1 \\
2 \\
3 \\
4 \\
5\end{array}$ & $\begin{array}{l}\text { biph_1 } \\
\text { biph_2 } \\
\text { biph_3 } \\
\text { biph_4 } \\
\text { biph_5 }\end{array}$ & & & & \\
\hline
\end{tabular}




\section{III.1 Structural parameters:}

Table S7. Selected $d_{1}$ bond distance ( $\AA$ ) (Table 1 in the manuscript) calculated (OPBE/cc-pVTZ) for all Chichibabin systems in CS, OSS singlet and T states.

\begin{tabular}{ccccccccccccc}
\hline $\boldsymbol{n}$ & State & $\mathbf{H}$ & OH & NH2 & CN & COH & Ph & FPh & NOPh & p_FPh & p_NO2Ph & BiPh \\
\hline & CS & 1.360 & 1.368 & 1.384 & 1.399 & 1.394 & 1.397 & 1.387 & 1.402 & 1.398 & 1.401 & 1.416 \\
$\mathbf{1}$ & OSS & 1.360 & 1.368 & 1.384 & 1.399 & 1.394 & 1.397 & 1.387 & 1.402 & 1.398 & 1.401 & 1.416 \\
& T & 1.434 & 1.418 & 1.439 & 1.458 & 1.434 & 1.467 & 1.460 & 1.467 & 1.467 & 1.467 & 1.461 \\
& CS & 1.367 & 1.376 & 1.395 & 1.410 & 1.408 & 1.411 & 1.400 & 1.419 & 1.412 & 1.418 & 1.429 \\
$\mathbf{2}$ & OSS & 1.367 & 1.376 & 1.395 & 1.410 & 1.408 & 1.411 & 1.400 & 1.425 & 1.412 & 1.418 & 1.447 \\
& T & 1.406 & 1.406 & 1.418 & 1.442 & 1.441 & 1.456 & 1.443 & 1.453 & 1.456 & 1.460 & 1.456 \\
& CS & 1.373 & 1.382 & 1.401 & 1.418 & 1.417 & 1.425 & 1.411 & 1.432 & 1.425 & 1.435 & 1.438 \\
$\mathbf{3}$ & OS & 1.393 & 1.390 & 1.402 & 1.431 & 1.439 & 1.448 & 1.432 & 1.444 & 1.448 & 1.451 & 1.453 \\
& T & 1.401 & 1.401 & 1.414 & 1.437 & 1.444 & 1.453 & 1.438 & 1.448 & 1.453 & 1.457 & 1.454 \\
& CS & 1.377 & 1.386 & 1.406 & 1.424 & 1.428 & 1.435 & 1.421 & 1.438 & 1.436 & 1.445 & 1.444 \\
$\mathbf{4}$ & OS & 1.398 & 1.399 & 1.412 & 1.435 & 1.436 & 1.451 & 1.436 & 1.445 & 1.451 & 1.456 & 1.454 \\
& T & 1.400 & 1.400 & 1.414 & 1.436 & 1.437 & 1.452 & 1.437 & 1.446 & 1.452 & 1.456 & 1.454 \\
& CS & 1.381 & 1.390 & 1.409 & 1.427 & 1.433 & 1.440 & 1.426 & 1.440 & 1.440 & 1.447 & 1.446 \\
$\mathbf{5}$ & OS & 1.399 & 1.400 & 1.413 & 1.435 & 1.436 & 1.451 & 1.436 & 1.446 & 1.451 & 1.455 & 1.454 \\
& T & 1.399 & 1.400 & 1.414 & 1.435 & 1.436 & 1.451 & 1.436 & 1.446 & 1.451 & 1.455 & 1.454 \\
\hline
\end{tabular}

Table S8. Selected $\mathrm{d}_{2}$ bond distance ( $\AA$ ) (Table 1 in the manuscript) calculated (OPBE/cc-pVTZ) for all Chichibabin systems in CS, OSS singlet and T states.

\begin{tabular}{ccccccccccccc}
\hline $\boldsymbol{n}$ & State & H & OH & NH2 & CN & COH & Ph & FPh & NOPh & p_FPh & p_NO2Ph & BiPh \\
\hline & CS & 1.352 & 1.355 & 1.359 & 1.359 & 1.358 & 1.361 & 1.356 & 1.360 & 1.361 & 1.362 & 1.370 \\
$\mathbf{1}$ & OSS & 1.352 & 1.355 & 1.359 & 1.359 & 1.358 & 1.361 & 1.356 & 1.362 & 1.361 & 1.362 & 1.370 \\
& T & 1.387 & 1.381 & 1.387 & 1.383 & 1.371 & 1.386 & 1.383 & 1.386 & 1.386 & 1.386 & 1.387 \\
& CS & 1.360 & 1.363 & 1.366 & 1.367 & 1.367 & 1.369 & 1.365 & 1.368 & 1.369 & 1.371 & 1.377 \\
$\mathbf{2}$ & OSS & 1.360 & 1.363 & 1.366 & 1.367 & 1.367 & 1.369 & 1.365 & 1.370 & 1.369 & 1.371 & 1.384 \\
& T & 1.381 & 1.381 & 1.381 & 1.382 & 1.378 & 1.385 & 1.383 & 1.382 & 1.385 & 1.385 & 1.387 \\
& CS & 1.365 & 1.367 & 1.369 & 1.372 & 1.372 & 1.375 & 1.371 & 1.374 & 1.375 & 1.378 & 1.380 \\
$\mathbf{3}$ & OSS & 1.375 & 1.371 & 1.369 & 1.377 & 1.380 & 1.383 & 1.379 & 1.380 & 1.383 & 1.384 & 1.386 \\
& T & 1.379 & 1.378 & 1.378 & 1.380 & 1.382 & 1.385 & 1.382 & 1.381 & 1.385 & 1.385 & 1.386 \\
& CS & 1.368 & 1.370 & 1.372 & 1.375 & 1.376 & 1.379 & 1.375 & 1.376 & 1.379 & 1.381 & 1.383 \\
$\mathbf{4}$ & OSS & 1.378 & 1.377 & 1.376 & 1.379 & 1.380 & 1.384 & 1.381 & 1.380 & 1.384 & 1.384 & 1.386 \\
& T & 1.378 & 1.378 & 1.378 & 1.379 & 1.381 & 1.381 & 1.381 & 1.381 & 1.384 & 1.385 & 1.386 \\
& CS & 1.371 & 1.372 & 1.374 & 1.376 & 1.378 & 1.381 & 1.377 & 1.377 & 1.381 & 1.382 & 1.384 \\
$\mathbf{5}$ & OSS & 1.378 & 1.377 & 1.377 & 1.379 & 1.381 & 1.384 & 1.381 & 1.379 & 1.384 & 1.384 & 1.386 \\
& T & 1.378 & 1.377 & 1.377 & 1.379 & 1.381 & 1.384 & 1.381 & 1.379 & 1.384 & 1.384 & 1.386 \\
\hline
\end{tabular}


Table S9. Selected $d_{3}$ bond distance $(\AA)$ (Table 1 in the manuscript) calculated (OPBE/cc-pVTZ) for all Chichibabin systems in CS, OSS singlet and T states.

\begin{tabular}{ccccccccccccc}
\hline $\boldsymbol{n}$ & State & H & OH & NH2 & CN & COH & Ph & FPh & NOPh & p_FPh & p_NO2Ph & BiPh \\
\hline \multirow{4}{*}{$\mathbf{2}$} & CS & 1.417 & 1.419 & 1.420 & 1.432 & 1.431 & 1.431 & 1.427 & 1.435 & 1.432 & 1.437 & 1.450 \\
& OSS & 1.418 & 1.419 & 1.420 & 1.432 & 1.431 & 1.431 & 1.427 & 1.442 & 1.432 & 1.437 & 1.468 \\
& T & 1.476 & 1.474 & 1.472 & 1.473 & 1.456 & 1.474 & 1.473 & 1.473 & 1.474 & 1.474 & 1.475 \\
& CS & 1.433 & 1.435 & 1.435 & 1.447 & 1.446 & 1.451 & 1.445 & 1.452 & 1.451 & 1.457 & 1.462 \\
$\mathbf{3}$ & OSS & 1.460 & 1.448 & 1.436 & 1.462 & 1.466 & 1.470 & 1.467 & 1.468 & 1.470 & 1.472 & 1.474 \\
& T & 1.472 & 1.470 & 1.467 & 1.471 & 1.471 & 1.474 & 1.473 & 1.472 & 1.474 & 1.474 & 1.475 \\
& CS & 1.446 & 1.446 & 1.446 & 1.456 & 1.458 & 1.462 & 1.458 & 1.459 & 1.463 & 1.467 & 1.469 \\
$\mathbf{4}$ & OSS & 1.468 & 1.465 & 1.460 & 1.468 & 1.469 & 1.473 & 1.471 & 1.469 & 1.473 & 1.474 & 1.475 \\
& T & 1.470 & 1.468 & 1.465 & 1.469 & 1.470 & 1.474 & 1.472 & 1.470 & 1.473 & 1.474 & 1.475 \\
& CS & 1.455 & 1.454 & 1.453 & 1.461 & 1.463 & 1.467 & 1.463 & 1.461 & 1.467 & 1.469 & 1.472 \\
$\mathbf{5}$ & OSS & 1.470 & 1.467 & 1.463 & 1.469 & 1.470 & 1.473 & 1.471 & 1.467 & 1.473 & 1.473 & 1.475 \\
& T & 1.470 & 1.467 & 1.464 & 1.469 & 1.470 & 1.473 & 1.471 & 1.468 & 1.473 & 1.473 & 1.475 \\
\hline
\end{tabular}

Table S10. Selected $d_{4}$ bond distance $(\AA)$ (Table 1 in the manuscript) calculated (OPBE/cc-pVTZ) for all Chichibabin systems in CS, OSS singlet and T states.

\begin{tabular}{ccccccccccccc}
\hline $\boldsymbol{n}$ & State & H & OH & NH2 & CN & COH & Ph & FPh & NOPh & p_FPh & p_NO2Ph & BiPh \\
\hline \multirow{3}{*}{$\mathbf{3}$} & CS & 1.372 & 1.373 & 1.374 & 1.376 & 1.376 & 1.378 & 1.376 & 1.377 & 1.378 & 1.380 & 1.383 \\
& OSS & 1.383 & 1.379 & 1.374 & 1.383 & 1.384 & 1.386 & 1.385 & 1.384 & 1.386 & 1.387 & 1.387 \\
& T & 1.387 & 1.387 & 1.387 & 1.386 & 1.386 & 1.388 & 1.387 & 1.386 & 1.388 & 1.387 & 1.388 \\
& CS & 1.378 & 1.379 & 1.379 & 1.380 & 1.381 & 1.384 & 1.382 & 1.381 & 1.384 & 1.385 & 1.386 \\
\multirow{4}{4}{} & OSS & 1.387 & 1.386 & 1.384 & 1.386 & 1.386 & 1.388 & 1.387 & 1.386 & 1.388 & 1.388 & 1.388 \\
& T & 1.387 & 1.387 & 1.387 & 1.386 & 1.387 & 1.388 & 1.387 & 1.386 & 1.388 & 1.388 & 1.388 \\
& CS & 1.382 & 1.382 & 1.382 & 1.383 & 1.384 & 1.386 & 1.385 & 1.383 & 1.386 & 1.386 & 1.387 \\
$\mathbf{5}$ & OSS & 1.387 & 1.387 & 1.386 & 1.386 & 1.387 & 1.388 & 1.387 & 1.386 & 1.388 & 1.388 & 1.388 \\
& T & 1.387 & 1.387 & 1.386 & 1.386 & 1.387 & 1.388 & 1.387 & 1.386 & 1.388 & 1.388 & 1.388 \\
\hline
\end{tabular}

Table S11. Selected $d_{5}$ bond distance $(\AA)$ (Table 1 in the manuscript) calculated (OPBE/cc-pVTZ) for all Chichibabin systems in CS, OSS singlet and T states.

\begin{tabular}{ccccccccccccc}
\hline & State & H & OH & NH2 & CN & COH & Ph & FPh & NOPh & p_FPh & p_NO2Ph & BiPh \\
\hline \multirow{4}{*}{4} & CS & 1.453 & 1.453 & 1.452 & 1.46 & 1.463 & 1.466 & 1.463 & 1.463 & 1.466 & 1.469 & 1.472 \\
& OSS & 1.474 & 1.471 & 1.467 & 1.473 & 1.474 & 1.475 & 1.475 & 1.473 & 1.475 & 1.476 & 1.477 \\
& T & 1.475 & 1.474 & 1.473 & 1.475 & 1.475 & 1.476 & 1.476 & 1.475 & 1.476 & 1.476 & 1.477 \\
& CS & 1.465 & 1.464 & 1.463 & 1.468 & 1.407 & 1.473 & 1.471 & 1.468 & 1.473 & 1.474 & 1.475 \\
\multirow{5}{*}{$\mathbf{5}$} & OSS & 1.476 & 1.474 & 1.473 & 1.475 & 1.475 & 1.476 & 1.476 & 1.474 & 1.476 & 1.477 & 1.477 \\
& T & 1.476 & 1.475 & 1.474 & 1.475 & 1.476 & 1.477 & 1.476 & 1.475 & 1.477 & 1.477 & 1.477 \\
\hline
\end{tabular}

Table S12. Selected $d_{6}$ bond distance $(\AA)$ (Table 1 in the manuscript) calculated (OPBE/cc-pVTZ) for all Chichibabin systems in CS, OSS singlet and T states.

\begin{tabular}{ccccccccccccc}
\hline $\boldsymbol{n}$ & State & H & OH & NH2 & CN & COH & Ph & FPh & NOPh & p_FPh & p_NO2Ph & BiPh \\
\hline \multirow{3}{*}{$\mathbf{5}$} & CS & 1.384 & 1.384 & 1.384 & 1.385 & 1.386 & 1.387 & 1.386 & 1.385 & 1.387 & 1.387 & 1.388 \\
& OSS & 1.388 & 1.388 & 1.387 & 1.387 & 1.388 & 1.388 & 1.388 & 1.387 & 1.388 & 1.388 & 1.388 \\
& T & 1.388 & 1.388 & 1.388 & 1.388 & 1.388 & 1.388 & 1.388 & 1.387 & 1.388 & 1.388 & 1.388 \\
\hline
\end{tabular}


Table S13. Selected $\alpha_{1}$ dihedral angle $\left(^{\circ}\right)$ (Table 1 in the manuscript) calculated (OPBE/cc-pVTZ) for all Chichibabin systems in CS, OSS singlet and T states.

\begin{tabular}{ccccccccccccc}
\hline $\boldsymbol{n}$ & State & H & OH & NH2 & CN & COH & Ph & FPh & NOPh & p_FPh & p_NO2Ph & BiPh \\
\hline \multirow{2}{*}{$\mathbf{2}$} & CS & -3.8 & 0.2 & -11.0 & 15.2 & 17.2 & 16.0 & 13.5 & -19.0 & -16.3 & -17.8 & -22.9 \\
& OSS & 3.8 & 0.2 & -11.0 & 15.2 & -13.8 & -16.1 & -13.5 & -21.8 & -16.3 & -17.8 & -34.0 \\
& T & -41.9 & 39.9 & 38.5 & 39.9 & -26.3 & 38.0 & 39.5 & -41.8 & 37.9 & 38.9 & -39.5 \\
& CS & 13.7 & -13.6 & 14.4 & -22.5 & 19.7 & 21.4 & 19.6 & 25.0 & -21.5 & -26.4 & 28.5 \\
$\mathbf{3}$ & OSS & -28.7 & -21.2 & 14.7 & -31.0 & 32.5 & -35.0 & -33.6 & -35.8 & -34.8 & -37.9 & 37.5 \\
& T & 36.9 & -34.8 & 32.7 & -37.2 & 36.2 & -38.4 & -37.9 & -39.8 & -38.4 & -39.3 & 38.9 \\
& CS & 20.0 & 20.0 & 17.5 & 25.9 & 26.2 & 28.4 & 26.9 & 28.7 & 28.5 & 32.1 & 33.4 \\
$\mathbf{4}$ & OSS & 33.8 & 30.5 & 26.5 & 33.8 & 34.3 & 36.8 & 35.6 & 35.9 & 36.8 & 37.3 & 38.5 \\
& T & 35.2 & 33.0 & 30.6 & 35.1 & 35.4 & 37.2 & 36.8 & 36.8 & 37.2 & 37.7 & 38.7 \\
& CS & 25.0 & 24.0 & 22.4 & 30.3 & 29.7 & 31.7 & 31.0 & 29.8 & 31.7 & 33.9 & 36.2 \\
$\mathbf{5}$ & OSS & 34.8 & 32.3 & 29.1 & 35.0 & 34.6 & 36.5 & 36.3 & 34.2 & 36.5 & 37.5 & 39.0 \\
& T & 34.9 & 32.7 & 29.9 & 34.7 & 34.8 & 36.6 & 36.4 & 34.5 & 36.6 & 37.6 & 39.0 \\
\hline
\end{tabular}

Table S14. Selected $\alpha_{2}$ dihedral angle $\left(^{\circ}\right)$ (Table 1 in the manuscript) calculated (OPBE/cc-pVTZ) for all Chichibabin systems in CS, OSS singlet and T states.

\begin{tabular}{|c|c|c|c|c|c|c|c|c|c|c|c|c|}
\hline$n$ & State & $\mathrm{H}$ & $\mathrm{OH}$ & $\mathrm{NH} 2$ & $\mathrm{CN}$ & $\mathrm{COH}$ & $\mathbf{P h}$ & FPh & NOPh & p_FPh & p_NO2Ph & $\mathrm{BiPh}$ \\
\hline \multirow{3}{*}{3} & $\mathrm{CS}$ & -13.7 & -13.6 & 14.4 & -22.6 & -19.7 & -21.4 & -19.6 & -25.0 & 21.5 & 26.4 & -29.6 \\
\hline & OSS & -28.7 & -21.2 & 14.7 & -31.0 & 31.9 & -35.0 & -33.6 & -35.8 & -34.8 & -37.9 & -38.4 \\
\hline & $\mathrm{T}$ & 36.9 & -34.8 & 32.7 & -37.2 & 35.9 & -38.4 & -37.9 & -39.8 & -38.4 & -39.3 & -39.4 \\
\hline \multirow{3}{*}{4} & $\mathrm{CS}$ & -23.4 & -23.2 & -22.5 & -28.0 & -29.3 & -31.5 & -29.1 & -30.4 & -31.5 & -33.9 & -36.1 \\
\hline & OSS & -37.2 & -34.9 & -31.7 & -37.0 & -37.7 & -39.0 & -38.8 & -37.4 & -39.0 & -39.8 & -40.0 \\
\hline & $\mathrm{T}$ & -39.0 & -37.9 & -36.7 & -38.9 & -39.1 & -39.5 & -39.5 & -38.8 & -39.5 & -40.2 & -40.2 \\
\hline \multirow{3}{*}{5} & $\mathrm{CS}$ & -30.8 & -30.2 & -28.8 & 33.8 & -34.9 & -36.9 & -35.0 & -34.7 & -36.9 & -37.5 & 38.5 \\
\hline & OSS & -38.9 & -37.8 & -36.3 & 39.2 & -39.6 & -40.2 & -39.7 & -39.3 & -40.2 & -39.9 & 40.5 \\
\hline & $\mathrm{T}$ & -40.0 & -38.3 & -37.4 & 39.6 & -39.9 & -40.3 & -39.9 & -39.7 & -40.2 & -40.0 & 40.5 \\
\hline
\end{tabular}

Table S15. Selected $\alpha_{3}$ dihedral angle $\left(^{\circ}\right)$ (Table 1 in the manuscript) calculated (OPBE/cc-pVTZ) for all Chichibabin systems in CS, OSS singlet and T states.

\begin{tabular}{ccccccccccccc}
\hline $\boldsymbol{n}$ & State & H & OH & NH2 & CN & COH & Ph & FPh & NOPh & p_FPh & p_NO2Ph & BiPh \\
\hline \multirow{4}{*}{4} & CS & 20.0 & 20.0 & 17.5 & 25.9 & 26.2 & 28.4 & 26.9 & 28.7 & 28.5 & 32.1 & -35.8 \\
& OSS & 33.8 & 30.7 & 26.6 & 33.8 & 34.3 & 36.8 & 35.6 & 35.9 & 36.8 & 37.3 & -39.5 \\
& T & 35.2 & 33.0 & 30.6 & 35.1 & 35.4 & 37.2 & 36.8 & 36.8 & 37.2 & 37.7 & -39.6 \\
& CS & 31.5 & 30.7 & 29.5 & -32.9 & -34.9 & -36.9 & -35.0 & -34.7 & -36.9 & -37.5 & 38.6 \\
\multirow{5}{*}{5} & OSS & 39.7 & 38.7 & 36.7 & -38.4 & -39.6 & -40.2 & -39.7 & -39.3 & -40.2 & -39.9 & 40.4 \\
& T & -40.0 & 39.2 & 37.8 & -38.9 & -39.9 & -40.3 & -39.9 & -39.7 & -40.2 & -40.0 & 40.4 \\
\hline
\end{tabular}

Table S16. Selected $\alpha_{4}$ dihedral angle $\left(^{\circ}\right)$ (Table 1 in the manuscript) calculated (OPBE/cc-pVTZ) for all Chichibabin systems in CS, OSS singlet and T states.

\begin{tabular}{ccccccccccccc}
\hline $\boldsymbol{n}$ & State & H & OH & NH2 & CN & COH & Ph & FPh & NOPh & p_FPh & p_NO2Ph & BiPh \\
\hline \multirow{4}{*}{5} & CS & 26.1 & 24.9 & 23.2 & 29.1 & 29.7 & 31.7 & 31.0 & 29.8 & 31.7 & 33.9 & 37.6 \\
& OSS & 35.3 & 32.8 & 29.5 & 34.4 & 34.6 & 36.5 & 36.3 & 34.2 & 36.5 & 37.5 & 39.7 \\
& T & 34.9 & 33.1 & 30.1 & 34.7 & 34.8 & 36.6 & 36.4 & 34.5 & 36.6 & 37.6 & 39.7 \\
\hline
\end{tabular}




\section{III.2 Energy values:}

Table S17. Calculated OPBE/cc-pVTZ relative energies in eV of CS, OSS and T states (referred to CS singlet) and Adiabatic $\Delta \mathrm{E}_{\mathrm{ST}}$ (where $\mathrm{S}$ is lowest singlet state, which can be CS or OSS) of all studied Chichibabin systems.

\begin{tabular}{|c|c|c|c|c|c|c|c|c|c|c|c|}
\hline \multirow{2}{*}{ system } & \multirow{2}{*}{$n$} & \multicolumn{3}{|c|}{ Electronic state } & \multirow{2}{*}{$\begin{array}{c}\text { Adiabatic } \\
\Delta \mathrm{E}_{\mathrm{ST}}\end{array}$} & \multirow{2}{*}{ system } & \multirow{2}{*}{$n$} & \multicolumn{3}{|c|}{ Electronic state } & \multirow{2}{*}{$\begin{array}{c}\text { Adiabatic } \\
\Delta \mathrm{E}_{\mathrm{ST}}\end{array}$} \\
\hline & & $\mathrm{CS}$ & OSS & $\mathrm{T}$ & & & & $\mathrm{CS}$ & OSS & $\mathrm{T}$ & \\
\hline \multirow{5}{*}{1} & 1 & 0.00 & 0.00 & 1.39 & 1.39 & \multirow{5}{*}{7} & 1 & 0.00 & 0.00 & 0.70 & 0.70 \\
\hline & 2 & 0.00 & 0.00 & 0.44 & 0.44 & & 2 & 0.00 & 0.00 & 0.19 & 0.19 \\
\hline & 3 & 0.00 & -0.09 & -0.02 & 0.07 & & 3 & 0.00 & -0.14 & -0.12 & 0.02 \\
\hline & 4 & 0.00 & -0.31 & -0.30 & 0.01 & & 4 & 0.00 & -0.27 & -0.26 & 0.00 \\
\hline & 5 & 0.00 & -0.43 & -0.43 & 0.00 & & 5 & 0.00 & -0.31 & -0.31 & 0.00 \\
\hline \multirow{5}{*}{2} & 1 & 0.00 & 0.00 & 1.32 & 1.32 & \multirow{5}{*}{8} & 1 & 0.00 & 0.00 & 0.70 & 0.70 \\
\hline & 2 & 0.00 & 0.00 & 0.54 & 0.54 & & 2 & 0.00 & 0.00 & 0.19 & 0.19 \\
\hline & 3 & 0.00 & -0.02 & 0.10 & 0.12 & & 3 & 0.00 & -0.13 & -0.11 & 0.02 \\
\hline & 4 & 0.00 & -0.17 & -0.16 & 0.02 & & 4 & 0.00 & -0.26 & -0.25 & 0.00 \\
\hline & 5 & 0.00 & -0.29 & -0.29 & 0.00 & & 5 & 0.00 & -0.30 & -0.30 & 0.00 \\
\hline \multirow{5}{*}{3} & 1 & 0.00 & 0.00 & 1.32 & 1.32 & \multirow{5}{*}{9} & 1 & 0.00 & 0.00 & 0.58 & 0.58 \\
\hline & 2 & 0.00 & 0.00 & 0.61 & 0.61 & & 2 & 0.00 & 0.00 & 0.12 & 0.12 \\
\hline & 3 & 0.00 & 0.00 & 0.19 & 0.19 & & 3 & 0.00 & -0.13 & -0.12 & 0.01 \\
\hline & 4 & 0.00 & -0.09 & -0.06 & 0.03 & & 4 & 0.00 & -0.22 & -0.22 & 0.00 \\
\hline & 5 & 0.00 & -0.19 & -0.18 & 0.01 & & 5 & 0.00 & -0.25 & -0.25 & 0.00 \\
\hline \multirow{5}{*}{4} & 1 & 0.00 & 0.00 & 0.40 & 0.40 & \multirow{5}{*}{10} & 1 & 0.00 & 0.00 & 0.87 & 0.87 \\
\hline & 2 & 0.00 & 0.00 & 0.16 & 0.16 & & 2 & 0.00 & 0.00 & 0.28 & 0.28 \\
\hline & 3 & 0.00 & -0.07 & -0.02 & 0.06 & & 3 & 0.00 & -0.11 & -0.07 & 0.04 \\
\hline & 4 & 0.00 & -0.21 & -0.21 & 0.01 & & 4 & 0.00 & -0.25 & -0.25 & 0.01 \\
\hline & 5 & 0.00 & -0.26 & -0.26 & 0.00 & & 5 & 0.00 & -0.32 & -0.31 & 0.00 \\
\hline \multirow{5}{*}{5} & 1 & 0.00 & 0.00 & 0.84 & 0.84 & \multirow{10}{*}{11} & 1 & 0.00 & 0.00 & 0.51 & 0.51 \\
\hline & 2 & 0.00 & 0.00 & 0.27 & 0.27 & & 2 & 0.00 & 0.00 & 0.13 & 0.13 \\
\hline & 3 & 0.00 & -0.08 & -0.02 & 0.06 & & 3 & 0.00 & -0.46 & -0.43 & 0.03 \\
\hline & 4 & 0.00 & -0.19 & -0.18 & 0.01 & & 4 & 0.00 & -0.53 & -0.52 & 0.01 \\
\hline & 5 & 0.00 & -0.26 & -0.25 & 0.00 & & 5 & 0.00 & -0.21 & -0.21 & 0.00 \\
\hline \multirow{5}{*}{6} & 1 & 0.00 & 0.00 & 0.30 & 0.30 & & & & & & \\
\hline & 2 & 0.00 & -0.06 & -0.02 & 0.04 & & & & & & \\
\hline & 3 & 0.00 & -0.19 & -0.19 & 0.01 & & & & & & \\
\hline & 4 & 0.00 & -0.26 & -0.26 & 0.00 & & & & & & \\
\hline & 5 & 0.00 & -0.29 & -0.29 & 0.00 & & & & & & \\
\hline
\end{tabular}

\section{III.3 $<\hat{\boldsymbol{S}}^{2}>$, Diradical character $(y)$ and Mulliken populations:}

Table S18. Calculated OPBE/cc-pVTZ $<\hat{S}^{2}>$ values for the OSS and T states of all studied Chichibabin systems.

\begin{tabular}{|c|c|c|c|c|c|c|c|c|c|c|c|}
\hline Structure & OSS & $T$ & Structure & OSS & $\mathbf{T}$ & Structure & OSS & $T$ & Structure & OSS & $\mathbf{T}$ \\
\hline h_1 & 0.00 & 2.01 & coh_1 & 0.00 & 2.01 & ph_1 & 0.00 & 2.02 & p_noph_1 & 0.00 & 2.02 \\
\hline h_2 & 0.00 & 2.03 & coh_2 & 0.00 & 2.01 & ph_2 & 0.00 & 2.02 & p_noph_2 & 0.00 & 2.02 \\
\hline h_3 & 0.87 & 2.03 & coh_3 & 0.81 & 2.02 & ph_3 & 0.96 & 2.02 & p_noph_3 & 1.00 & 2.02 \\
\hline h_4 & 1.02 & 2.03 & coh_4 & 1.00 & 2.02 & ph_4 & 1.02 & 2.02 & p_noph_4 & 1.01 & 2.02 \\
\hline h_ 5 & 1.03 & 2.03 & $\operatorname{coh} 5$ & 1.01 & 2.02 & ph_5 & 1.02 & 2.02 & p_noph_5 & 1.02 & 2.02 \\
\hline oh_1 & 0.00 & 2.01 & cn_1 & 0.00 & 2.02 & fph_1 & 0.00 & 2.01 & noph_1 & 0.00 & 2.02 \\
\hline oh_2 & 0.00 & 2.02 & cn_2 & 0.00 & 2.02 & fph_2 & 0.00 & 2.02 & noph_2 & 0.27 & 2.02 \\
\hline oh_3 & 0.56 & 2.02 & cn_3 & 0.83 & 2.02 & fph_3 & 0.92 & 2.02 & noph_3 & 0.91 & 2.02 \\
\hline oh_- 4 & 0.89 & 2.02 & cn_4 & 0.99 & 2.02 & fph_4 & 1.01 & 2.02 & noph_4 & 0.99 & 2.02 \\
\hline oh_5 & 1.02 & 2.02 & cn_5 & 1.01 & 2.02 & fph 5 & 1.02 & 2.02 & noph_5 & 1.01 & 2.02 \\
\hline nh_1 & 0.00 & 2.01 & biph_1 & 0.00 & 2.02 & p_fph_1 & 0.00 & 2.02 & & & \\
\hline nh_2 & 0.00 & 2.01 & biph_2 & 0.85 & 2.02 & p_fph_2 & 0.00 & 2.02 & & & \\
\hline nh_3 & 0.03 & 2.01 & biph_3 & 1.00 & 2.02 & p_fph_3 & 0.96 & 2.02 & & & \\
\hline nh_4 & 0.88 & 2.02 & biph_4 & 1.02 & 2.02 & p_fph_4 & 1.01 & 2.02 & & & \\
\hline nh_5 & 0.99 & 2.02 & biph_5 & 1.02 & 2.02 & p_fph_5 & 1.02 & 2.02 & & & \\
\hline
\end{tabular}


Table S19. Calculated OPBE/cc-pVTZ HOMO and LUMO natural orbitals occupations ( $n_{\mathrm{HOMO}}$ and $\left.n_{\mathrm{LUMO}}\right)$ diradical character $(y)$ values for the OSS and T states of all studied Chichibabin systems.

\begin{tabular}{|c|c|c|c|c|c|c|}
\hline \multirow{2}{*}{ Structure } & \multicolumn{3}{|c|}{$\overline{\text { OSS }}$} & \multicolumn{3}{|c|}{$\bar{T}$} \\
\hline & $n_{\mathrm{HOMO}}$ & $n_{\text {LUMO }}$ & $y$ & $n_{\mathrm{HOMO}}$ & $n_{\text {LUMO }}$ & $y$ \\
\hline h 1 & 2.00 & 0.00 & 0.00 & 1.00 & 1.00 & 1.00 \\
\hline h_2 & 2.00 & 0.00 & 0.00 & 1.00 & 1.00 & 1.00 \\
\hline h_3 & 1.40 & 0.60 & 0.31 & 1.00 & 1.00 & 1.00 \\
\hline h_4 & 1.13 & 0.87 & 0.75 & 1.00 & 1.00 & 1.00 \\
\hline h_5 & 1.05 & 0.95 & 0.90 & 1.00 & 1.00 & 1.00 \\
\hline oh_1 & 2.00 & 0.00 & 0.00 & 1.00 & 1.00 & 1.00 \\
\hline oh 2 & 2.00 & 0.00 & 0.00 & 1.00 & 1.00 & 1.00 \\
\hline oh_3 & 1.67 & 0.33 & 0.07 & 1.00 & 1.00 & 1.00 \\
\hline oh_4 & 1.22 & 0.78 & 0.58 & 1.00 & 1.00 & 1.00 \\
\hline oh 5 & 1.09 & 0.91 & 0.83 & 1.00 & 1.00 & 1.00 \\
\hline nh 1 & 2.00 & 0.00 & 0.00 & 1.00 & 1.00 & 1.00 \\
\hline $\mathrm{nh}^{-} 2$ & 2.00 & 0.00 & 0.00 & 1.00 & 1.00 & 1.00 \\
\hline nh_3 & 1.98 & 0.02 & 0.00 & 1.00 & 1.00 & 1.00 \\
\hline nh_4 & 1.36 & 0.64 & 0.36 & 1.00 & 1.00 & 1.00 \\
\hline$n h^{-} 5$ & 1.16 & 0.84 & 0.70 & 1.00 & 1.00 & 1.00 \\
\hline cn_1 & 2.00 & 0.00 & 0.00 & 1.00 & 1.00 & 1.00 \\
\hline cn_2 & 2.00 & 0.00 & 0.00 & 1.00 & 1.00 & 1.00 \\
\hline cn_3 & 1.44 & 0.56 & 0.26 & 1.00 & 1.00 & 1.00 \\
\hline cn_4 & 1.18 & 0.82 & 0.64 & 1.00 & 1.00 & 1.00 \\
\hline $\mathrm{cn}^{-} 5$ & 1.09 & 0.91 & 0.82 & 1.00 & 1.00 & 1.00 \\
\hline coh_1 & 2.00 & 0.00 & 0.00 & 1.00 & 1.00 & 1.00 \\
\hline $\operatorname{coh} 2$ & 2.00 & 0.00 & 0.00 & 1.00 & 1.00 & 1.00 \\
\hline coh_3 & 1.35 & 0.65 & 0.38 & 1.00 & 1.00 & 1.00 \\
\hline $\operatorname{coh} 4$ & 1.15 & 0.86 & 0.72 & 1.00 & 1.00 & 1.00 \\
\hline $\operatorname{coh} 5$ & 1.08 & 0.92 & 0.85 & 1.00 & 1.00 & 1.00 \\
\hline ph_1 & 2.00 & 0.00 & 0.00 & 1.00 & 1.00 & 1.00 \\
\hline ph_2 & 2.00 & 0.00 & 0.00 & 1.00 & 1.00 & 1.00 \\
\hline ph_3 & 1.25 & 0.75 & 0.53 & 1.00 & 1.00 & 1.00 \\
\hline ph_4 & 1.09 & 0.91 & 0.83 & 1.00 & 1.00 & 1.00 \\
\hline ph 5 & 1.04 & 0.96 & 0.93 & 1.00 & 1.00 & 1.00 \\
\hline fph_1 & 2.00 & 0.00 & 0.00 & 1.00 & 1.00 & 1.00 \\
\hline fph_2 & 2.00 & 0.00 & 0.00 & 1.00 & 1.00 & 1.00 \\
\hline fph_3 & 1.32 & 0.68 & 0.42 & 1.00 & 1.00 & 1.00 \\
\hline fph_4 & 1.11 & 0.89 & 0.78 & 1.00 & 1.00 & 1.00 \\
\hline fph 5 & 1.05 & 0.95 & 0.90 & 1.00 & 1.00 & 1.00 \\
\hline noph_1 & 2.00 & 0.00 & 0.00 & 1.00 & 1.00 & 1.00 \\
\hline noph_2 & 1.86 & 0.14 & 0.01 & 1.00 & 1.00 & 1.00 \\
\hline noph_3 & 1.32 & 0.68 & 0.41 & 1.00 & 1.00 & 1.00 \\
\hline noph 4 & 1.18 & 0.82 & 0.66 & 1.00 & 1.00 & 1.00 \\
\hline noph 5 & 1.11 & 0.89 & 0.79 & 1.00 & 1.00 & 1.00 \\
\hline p_fph_1 & 2.00 & 0.00 & 0.00 & 1.00 & 1.00 & 1.00 \\
\hline p_fph_2 & 2.00 & 0.00 & 0.00 & 1.00 & 1.00 & 1.00 \\
\hline p_fph_3 & 1.26 & 0.74 & 0.52 & 1.00 & 1.00 & 1.00 \\
\hline p_fph_4 & 1.09 & 0.91 & 0.82 & 1.00 & 1.00 & 1.00 \\
\hline p_fph 5 & 1.04 & 0.96 & 0.93 & 1.00 & 1.00 & 1.00 \\
\hline p_noph_1 & 2.00 & 0.00 & 0.00 & 1.00 & 1.00 & 1.00 \\
\hline p_noph_2 & 2.00 & 0.00 & 0.00 & 1.00 & 1.00 & 1.00 \\
\hline p_noph_3 & 1.22 & 0.78 & 0.57 & 1.00 & 1.00 & 1.00 \\
\hline p_noph_4 & 1.08 & 0.92 & 0.83 & 1.00 & 1.00 & 1.00 \\
\hline p noph 5 & 1.04 & 0.96 & 0.93 & 1.00 & 1.00 & 1.00 \\
\hline biph_1 & 2.00 & 0.00 & 0.00 & 1.00 & 1.00 & 1.00 \\
\hline biph 2 & 1.41 & 0.59 & 0.29 & 1.00 & 1.00 & 1.00 \\
\hline biph_3 & 1.14 & 0.86 & 0.73 & 1.00 & 1.00 & 1.00 \\
\hline biph_4 & 1.06 & 0.94 & 0.89 & 1.00 & 1.00 & 1.00 \\
\hline biph_5 & 1.02 & 0.98 & 0.95 & 1.00 & 1.00 & 1.00 \\
\hline
\end{tabular}




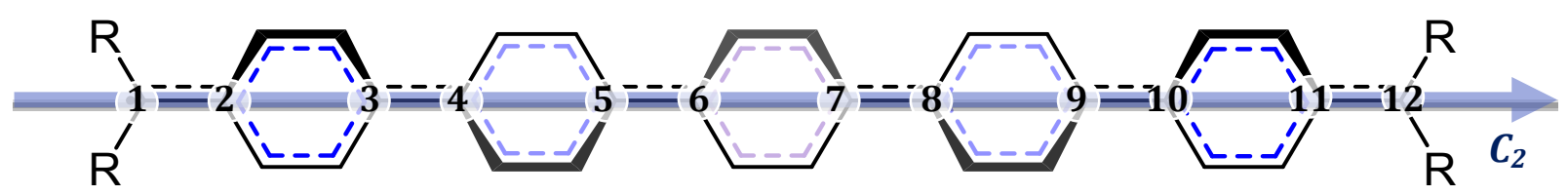

Figure S4. Numbered Carbons included in the $\mathrm{C}_{2}$ symmetry axis of the Chichibabin systems.

Table S20. Computed Mulliken Charge (Spin) densities of the selected carbons in figure S4 for the CS, OSS and T (OSS and T) states of R=H Chichibabin systems, at OPBE/cc-pVTZ level of theory.

\begin{tabular}{|c|c|c|c|c|c|c|}
\hline \multirow{2}{*}{ Structure } & \multirow{2}{*}{ Atom } & \multirow{2}{*}{$\begin{array}{c}\text { CS } \\
\text { Charge }\end{array}$} & \multicolumn{2}{|c|}{ OSS } & \multicolumn{2}{|c|}{$\mathbf{T}$} \\
\hline & & & Charge & Spin & Charge & Spin \\
\hline \multirow{4}{*}{ h_1 } & 1 & -0.18 & -0.18 & 0.00 & -0.18 & 0.87 \\
\hline & 2 & 0.03 & 0.03 & 0.00 & 0.04 & 0.02 \\
\hline & 3 & 0.03 & 0.03 & 0.00 & 0.04 & 0.02 \\
\hline & 4 & -0.18 & -0.18 & 0.00 & -0.18 & 0.87 \\
\hline \multirow{6}{*}{ h_2 } & 1 & -0.18 & -0.18 & 0.00 & -0.18 & 0.74 \\
\hline & 2 & 0.03 & 0.03 & 0.00 & 0.04 & -0.09 \\
\hline & 3 & 0.10 & 0.10 & 0.00 & 0.06 & 0.19 \\
\hline & 4 & 0.10 & 0.10 & 0.00 & 0.06 & 0.19 \\
\hline & 5 & 0.03 & 0.03 & 0.00 & 0.04 & -0.09 \\
\hline & 6 & -0.18 & -0.18 & 0.00 & -0.18 & 0.74 \\
\hline \multirow{8}{*}{ h_3 } & 1 & -0.18 & -0.18 & -0.62 & -0.18 & 0.71 \\
\hline & 2 & 0.04 & 0.04 & 0.10 & 0.04 & -0.10 \\
\hline & 3 & 0.07 & 0.06 & -0.21 & 0.06 & 0.21 \\
\hline & 4 & 0.07 & 0.06 & 0.08 & 0.06 & 0.00 \\
\hline & 5 & 0.07 & 0.06 & -0.08 & 0.06 & 0.00 \\
\hline & 6 & 0.07 & 0.06 & 0.21 & 0.06 & 0.21 \\
\hline & 7 & 0.04 & 0.04 & -0.10 & 0.04 & -0.10 \\
\hline & 8 & -0.18 & -0.18 & 0.62 & -0.18 & 0.71 \\
\hline \multirow{10}{*}{ h_4 } & 1 & -0.18 & -0.18 & 0.69 & -0.18 & 0.70 \\
\hline & 2 & 0.04 & 0.04 & -0.10 & 0.04 & -0.10 \\
\hline & 3 & 0.06 & 0.06 & 0.22 & 0.06 & 0.22 \\
\hline & 4 & 0.07 & 0.06 & -0.04 & 0.06 & -0.03 \\
\hline & 5 & 0.06 & 0.06 & 0.05 & 0.06 & 0.04 \\
\hline & 6 & 0.06 & 0.06 & -0.05 & 0.06 & 0.04 \\
\hline & 7 & 0.07 & 0.06 & 0.04 & 0.06 & -0.03 \\
\hline & 8 & 0.06 & 0.06 & -0.22 & 0.06 & 0.22 \\
\hline & 9 & 0.04 & 0.04 & 0.10 & 0.04 & -0.10 \\
\hline & 10 & -0.18 & -0.18 & -0.69 & -0.18 & 0.70 \\
\hline \multirow{12}{*}{ h_5 } & 1 & -0.19 & -0.18 & -0.69 & -0.18 & 0.70 \\
\hline & 2 & 0.04 & 0.04 & 0.10 & 0.04 & -0.10 \\
\hline & 3 & 0.06 & 0.06 & -0.22 & 0.06 & 0.22 \\
\hline & 4 & 0.06 & 0.06 & 0.03 & 0.06 & -0.03 \\
\hline & 5 & 0.06 & 0.06 & -0.04 & 0.06 & 0.04 \\
\hline & 6 & 0.06 & 0.06 & 0.01 & 0.06 & 0.00 \\
\hline & 7 & 0.06 & 0.06 & -0.01 & 0.06 & 0.00 \\
\hline & 8 & 0.06 & 0.06 & 0.04 & 0.06 & 0.04 \\
\hline & 9 & 0.06 & 0.06 & -0.03 & 0.06 & -0.03 \\
\hline & 10 & 0.06 & 0.06 & 0.22 & 0.06 & 0.22 \\
\hline & 11 & 0.04 & 0.04 & -0.10 & 0.04 & -0.10 \\
\hline & 12 & -0.19 & -0.18 & 0.69 & -0.18 & 0.70 \\
\hline
\end{tabular}


Table S21. Computed Mulliken Charge (Spin) densities of the selected carbons in figure S4 for the CS, OSS and T (OSS and T) states of R=OH Chichibabin systems, at OPBE/cc-pVTZ level of theory.

\begin{tabular}{|c|c|c|c|c|c|c|}
\hline \multirow{2}{*}{ Structure } & \multirow{2}{*}{ Atom } & \multirow{2}{*}{$\begin{array}{c}\text { CS } \\
\text { Charge }\end{array}$} & \multicolumn{2}{|c|}{ OSS } & \multicolumn{2}{|c|}{$\mathbf{T}$} \\
\hline & & & Charge & Spin & Charge & Spin \\
\hline \multirow{4}{*}{ oh_1 } & 1 & 0.18 & 0.18 & 0.00 & 0.18 & 0.62 \\
\hline & 2 & 0.00 & 0.00 & 0.00 & 0.00 & 0.10 \\
\hline & 3 & 0.00 & 0.00 & 0.00 & 0.00 & 0.06 \\
\hline & 4 & 0.18 & 0.18 & 0.00 & 0.19 & 0.49 \\
\hline \multirow{6}{*}{ oh_2 } & 1 & 0.19 & 0.19 & 0.00 & 0.18 & 0.48 \\
\hline & 2 & 0.00 & 0.00 & 0.00 & 0.03 & -0.03 \\
\hline & 3 & 0.09 & 0.09 & 0.00 & 0.05 & 0.19 \\
\hline & 4 & 0.09 & 0.09 & 0.00 & 0.05 & 0.19 \\
\hline & 5 & 0.00 & 0.00 & 0.00 & 0.03 & -0.03 \\
\hline & 6 & 0.19 & 0.19 & 0.00 & 0.17 & 0.48 \\
\hline \multirow{8}{*}{ oh_3 } & 1 & 0.19 & 0.19 & -0.31 & 0.18 & 0.45 \\
\hline & 2 & 0.01 & 0.01 & 0.04 & 0.02 & -0.04 \\
\hline & 3 & 0.07 & 0.05 & -0.17 & 0.05 & 0.21 \\
\hline & 4 & 0.06 & 0.06 & 0.08 & 0.06 & 0.01 \\
\hline & 5 & 0.06 & 0.06 & -0.08 & 0.06 & 0.01 \\
\hline & 6 & 0.07 & 0.05 & 0.17 & 0.05 & 0.21 \\
\hline & 7 & 0.01 & 0.01 & -0.04 & 0.02 & -0.04 \\
\hline & 8 & 0.19 & 0.19 & 0.31 & 0.18 & 0.45 \\
\hline \multirow{10}{*}{ oh_4 } & 1 & 0.19 & 0.18 & 0.42 & 0.18 & 0.44 \\
\hline & 2 & 0.01 & 0.02 & -0.04 & 0.02 & -0.04 \\
\hline & 3 & 0.05 & 0.05 & 0.22 & 0.05 & 0.22 \\
\hline & 4 & 0.06 & 0.06 & -0.04 & 0.06 & -0.02 \\
\hline & 5 & 0.05 & 0.06 & 0.06 & 0.06 & 0.04 \\
\hline & 6 & 0.05 & 0.06 & -0.06 & 0.06 & 0.04 \\
\hline & 7 & 0.06 & 0.06 & 0.04 & 0.06 & -0.02 \\
\hline & 8 & 0.05 & 0.05 & -0.22 & 0.05 & 0.22 \\
\hline & 9 & 0.01 & 0.02 & 0.04 & 0.02 & -0.04 \\
\hline & 10 & 0.19 & 0.18 & -0.42 & 0.18 & 0.44 \\
\hline \multirow{12}{*}{ oh_5 } & 1 & 0.19 & 0.18 & -0.43 & 0.18 & 0.43 \\
\hline & 2 & 0.01 & 0.02 & 0.04 & 0.02 & -0.04 \\
\hline & 3 & 0.05 & 0.05 & -0.22 & 0.05 & 0.22 \\
\hline & 4 & 0.06 & 0.06 & 0.03 & 0.06 & -0.03 \\
\hline & 5 & 0.05 & 0.06 & -0.05 & 0.06 & 0.05 \\
\hline & 6 & 0.06 & 0.06 & 0.02 & 0.06 & 0.00 \\
\hline & 7 & 0.06 & 0.06 & -0.02 & 0.06 & 0.00 \\
\hline & 8 & 0.05 & 0.06 & 0.05 & 0.06 & 0.05 \\
\hline & 9 & 0.06 & 0.06 & -0.03 & 0.06 & -0.03 \\
\hline & 10 & 0.05 & 0.05 & 0.22 & 0.05 & 0.22 \\
\hline & 11 & 0.01 & 0.02 & -0.04 & 0.02 & -0.04 \\
\hline & 12 & 0.19 & 0.18 & 0.43 & 0.18 & 0.43 \\
\hline
\end{tabular}


Table S22. Computed Mulliken Charge (Spin) densities of the selected carbons in figure S4 for the CS, OSS and $\mathrm{T}$ (OSS and T) states of $\mathrm{R}=\mathrm{NH}_{2}$ Chichibabin systems, at OPBE/cc-pVTZ level of theory.

\begin{tabular}{|c|c|c|c|c|c|c|}
\hline \multirow{2}{*}{ Structure } & \multirow{2}{*}{ Atom } & \multirow{2}{*}{$\begin{array}{c}\text { CS } \\
\text { Charge }\end{array}$} & \multicolumn{2}{|c|}{ OSS } & \multicolumn{2}{|c|}{$\mathbf{T}$} \\
\hline & & & Charge & Spin & Charge & Spin \\
\hline \multirow{4}{*}{ nh_1 } & 1 & 0.06 & 0.06 & 0.00 & 0.05 & 0.51 \\
\hline & 2 & 0.04 & 0.04 & 0.00 & 0.07 & 0.09 \\
\hline & 3 & 0.04 & 0.04 & 0.00 & 0.07 & 0.09 \\
\hline & 4 & 0.06 & 0.06 & 0.00 & 0.05 & 0.51 \\
\hline \multirow{6}{*}{ nh_2 } & 1 & 0.06 & 0.06 & 0.00 & 0.051 & 0.383 \\
\hline & 2 & 0.07 & 0.07 & 0.00 & 0.099 & 0.014 \\
\hline & 3 & 0.06 & 0.06 & 0.00 & 0.043 & 0.190 \\
\hline & 4 & 0.06 & 0.06 & 0.00 & 0.043 & 0.190 \\
\hline & 5 & 0.07 & 0.07 & 0.00 & 0.099 & 0.014 \\
\hline & 6 & 0.06 & 0.06 & 0.00 & 0.051 & 0.383 \\
\hline \multirow{8}{*}{ nh_3 } & 1 & 0.06 & 0.06 & -0.05 & 0.06 & 0.35 \\
\hline & 2 & 0.08 & 0.08 & 0.00 & 0.09 & 0.01 \\
\hline & 3 & 0.06 & 0.06 & -0.04 & 0.04 & 0.21 \\
\hline & 4 & 0.06 & 0.06 & 0.02 & 0.06 & 0.02 \\
\hline & 5 & 0.06 & 0.06 & -0.02 & 0.06 & 0.02 \\
\hline & 6 & 0.06 & 0.06 & 0.04 & 0.04 & 0.21 \\
\hline & 7 & 0.08 & 0.08 & 0.00 & 0.09 & 0.01 \\
\hline & 8 & 0.06 & 0.06 & 0.05 & 0.06 & 0.35 \\
\hline \multirow{10}{*}{ nh_4 } & 1 & 0.06 & 0.06 & -0.30 & 0.06 & 0.33 \\
\hline & 2 & 0.08 & 0.09 & -0.01 & 0.10 & 0.01 \\
\hline & 3 & 0.06 & 0.06 & 0.04 & 0.06 & -0.02 \\
\hline & 4 & 0.05 & 0.04 & -0.20 & 0.04 & 0.21 \\
\hline & 5 & 0.05 & 0.05 & -0.07 & 0.05 & 0.05 \\
\hline & 6 & 0.05 & 0.05 & 0.07 & 0.05 & 0.05 \\
\hline & 7 & 0.06 & 0.06 & -0.04 & 0.06 & -0.02 \\
\hline & 8 & 0.05 & 0.04 & 0.20 & 0.04 & 0.21 \\
\hline & 9 & 0.08 & 0.09 & 0.01 & 0.10 & 0.01 \\
\hline & 10 & 0.06 & 0.06 & 0.30 & 0.06 & 0.33 \\
\hline \multirow{12}{*}{ nh_5 } & 1 & 0.06 & 0.06 & -0.32 & 0.06 & 0.32 \\
\hline & 2 & 0.09 & 0.10 & -0.01 & 0.10 & 0.01 \\
\hline & 3 & 0.04 & 0.04 & -0.21 & 0.04 & 0.21 \\
\hline & 4 & 0.06 & 0.06 & 0.03 & 0.06 & -0.02 \\
\hline & 5 & 0.05 & 0.05 & -0.06 & 0.06 & 0.06 \\
\hline & 6 & 0.05 & 0.06 & 0.02 & 0.06 & 0.00 \\
\hline & 7 & 0.05 & 0.06 & -0.02 & 0.06 & 0.00 \\
\hline & 8 & 0.05 & 0.05 & 0.06 & 0.05 & 0.06 \\
\hline & 9 & 0.06 & 0.06 & -0.03 & 0.06 & -0.02 \\
\hline & 10 & 0.04 & 0.04 & 0.21 & 0.04 & 0.21 \\
\hline & 11 & 0.09 & 0.10 & 0.01 & 0.10 & 0.01 \\
\hline & 12 & 0.06 & 0.06 & 0.32 & 0.06 & 0.32 \\
\hline
\end{tabular}


Table S23. Computed Mulliken Charge (Spin) densities of the selected carbons in figure S4 for the CS, OSS and T (OSS and T) states of $\mathrm{R}=\mathrm{COH}$ Chichibabin systems, at OPBE/cc-pVTZ level of theory.

\begin{tabular}{|c|c|c|c|c|c|c|}
\hline \multirow{2}{*}{ Structure } & \multirow{2}{*}{ Atom } & \multirow{2}{*}{$\begin{array}{c}\text { CS } \\
\text { Charge }\end{array}$} & \multicolumn{2}{|c|}{ OSS } & \multicolumn{2}{|c|}{$T$} \\
\hline & & & Charge & Spin & Charge & Spin \\
\hline \multirow{4}{*}{ coh_1 } & 1 & 0.03 & 0.03 & 0.00 & -0.02 & 0.25 \\
\hline & 2 & 0.05 & 0.05 & 0.00 & 0.09 & 0.08 \\
\hline & 3 & 0.05 & 0.05 & 0.00 & 0.09 & 0.08 \\
\hline & 4 & 0.03 & 0.03 & 0.00 & -0.02 & 0.25 \\
\hline \multirow{6}{*}{ coh_2 } & 1 & 0.00 & 0.00 & 0.00 & -0.06 & 0.23 \\
\hline & 2 & 0.06 & 0.06 & 0.00 & 0.08 & 0.03 \\
\hline & 3 & 0.08 & 0.09 & 0.00 & 0.06 & 0.07 \\
\hline & 4 & 0.08 & 0.09 & 0.00 & 0.07 & 0.08 \\
\hline & 5 & 0.06 & 0.06 & 0.00 & 0.08 & 0.03 \\
\hline & 6 & 0.00 & 0.00 & 0.00 & -0.06 & 0.25 \\
\hline \multirow{8}{*}{ coh_3 } & 1 & -0.01 & -0.02 & 0.38 & -0.01 & 0.42 \\
\hline & 2 & 0.06 & 0.08 & -0.03 & 0.08 & -0.01 \\
\hline & 3 & 0.07 & 0.06 & 0.10 & 0.06 & 0.10 \\
\hline & 4 & 0.07 & 0.06 & -0.04 & 0.06 & 0.02 \\
\hline & 5 & 0.07 & 0.06 & 0.03 & 0.06 & 0.01 \\
\hline & 6 & 0.07 & 0.07 & -0.12 & 0.07 & 0.14 \\
\hline & 7 & 0.06 & 0.07 & 0.04 & 0.05 & -0.05 \\
\hline & 8 & -0.01 & -0.04 & -0.33 & 0.00 & 0.47 \\
\hline \multirow{10}{*}{ coh_4 } & 1 & -0.02 & 0.01 & 0.49 & 0.01 & 0.50 \\
\hline & 2 & 0.07 & 0.05 & -0.06 & 0.05 & -0.06 \\
\hline & 3 & 0.07 & 0.07 & 0.15 & 0.07 & 0.15 \\
\hline & 4 & 0.06 & 0.06 & -0.02 & 0.06 & -0.01 \\
\hline & 5 & 0.06 & 0.06 & 0.04 & 0.06 & 0.03 \\
\hline & 6 & 0.06 & 0.06 & -0.04 & 0.06 & 0.03 \\
\hline & 7 & 0.06 & 0.06 & 0.02 & 0.06 & -0.01 \\
\hline & 8 & 0.07 & 0.07 & -0.15 & 0.07 & 0.15 \\
\hline & 9 & 0.07 & 0.05 & 0.06 & 0.05 & -0.06 \\
\hline & 10 & -0.02 & 0.01 & -0.49 & 0.01 & 0.50 \\
\hline \multirow{12}{*}{ coh_5 } & 1 & -0.03 & 0.01 & -0.49 & 0.01 & 0.50 \\
\hline & 2 & 0.07 & 0.05 & 0.06 & 0.05 & -0.06 \\
\hline & 3 & 0.07 & 0.07 & -0.15 & 0.07 & 0.15 \\
\hline & 4 & 0.06 & 0.06 & 0.02 & 0.06 & -0.01 \\
\hline & 5 & 0.06 & 0.06 & -0.04 & 0.06 & 0.03 \\
\hline & 6 & 0.06 & 0.06 & 0.01 & 0.06 & 0.00 \\
\hline & 7 & 0.06 & 0.06 & -0.01 & 0.06 & 0.00 \\
\hline & 8 & 0.06 & 0.06 & 0.04 & 0.06 & 0.03 \\
\hline & 9 & 0.06 & 0.06 & -0.02 & 0.06 & -0.01 \\
\hline & 10 & 0.07 & 0.07 & 0.15 & 0.07 & 0.15 \\
\hline & 11 & 0.07 & 0.05 & -0.06 & 0.05 & -0.06 \\
\hline & 12 & -0.03 & 0.01 & 0.49 & 0.01 & 0.50 \\
\hline
\end{tabular}


Table S24. Computed Mulliken Charge (Spin) densities of the selected carbons in figure S4 for the CS, OSS and T (OSS and T) states of $\mathrm{R}=\mathrm{CN}$ Chichibabin systems, at OPBE/cc-pVTZ level of theory.

\begin{tabular}{|c|c|c|c|c|c|c|}
\hline \multirow{2}{*}{ Structure } & \multirow{2}{*}{ Atom } & \multirow{2}{*}{$\begin{array}{c}\text { CS } \\
\text { Charge }\end{array}$} & \multicolumn{2}{|c|}{ OSS } & \multicolumn{2}{|c|}{$\mathbf{T}$} \\
\hline & & & Charge & Spin & Charge & Spin \\
\hline \multirow{4}{*}{ cn_1 } & 1 & 0.17 & 0.17 & 0.00 & 0.17 & 0.51 \\
\hline & 2 & 0.02 & 0.02 & 0.00 & 0.01 & 0.05 \\
\hline & 3 & 0.02 & 0.02 & 0.00 & 0.01 & 0.05 \\
\hline & 4 & 0.17 & 0.17 & 0.00 & 0.17 & 0.51 \\
\hline \multirow{6}{*}{ cn_2 } & 1 & 0.15 & 0.15 & 0.00 & 0.15 & 0.48 \\
\hline & 2 & 0.02 & 0.02 & 0.00 & 0.01 & -0.03 \\
\hline & 3 & 0.08 & 0.08 & 0.00 & 0.07 & 0.14 \\
\hline & 4 & 0.08 & 0.08 & 0.00 & 0.07 & 0.14 \\
\hline & 5 & 0.02 & 0.02 & 0.00 & 0.01 & -0.03 \\
\hline & 6 & 0.15 & 0.15 & 0.00 & 0.15 & 0.48 \\
\hline \multirow{8}{*}{ cn_3 } & 1 & 0.13 & 0.14 & 0.40 & 0.15 & 0.46 \\
\hline & 2 & 0.02 & 0.02 & -0.05 & 0.01 & -0.04 \\
\hline & 3 & 0.07 & 0.06 & 0.15 & 0.07 & 0.16 \\
\hline & 4 & 0.07 & 0.07 & -0.06 & 0.06 & 0.01 \\
\hline & 5 & 0.07 & 0.07 & 0.06 & 0.06 & 0.01 \\
\hline & 6 & 0.07 & 0.06 & -0.15 & 0.07 & 0.16 \\
\hline & 7 & 0.02 & 0.02 & 0.05 & 0.01 & -0.04 \\
\hline & 8 & 0.13 & 0.14 & -0.40 & 0.15 & 0.46 \\
\hline \multirow{10}{*}{ cn_4 } & 1 & 0.13 & 0.14 & 0.44 & 0.15 & 0.45 \\
\hline & 2 & 0.02 & 0.02 & -0.04 & 0.02 & -0.04 \\
\hline & 3 & 0.06 & 0.07 & 0.16 & 0.07 & 0.16 \\
\hline & 4 & 0.07 & 0.06 & -0.03 & 0.06 & -0.01 \\
\hline & 5 & 0.06 & 0.06 & 0.05 & 0.06 & 0.03 \\
\hline & 6 & 0.06 & 0.06 & -0.05 & 0.06 & 0.03 \\
\hline & 7 & 0.07 & 0.06 & 0.03 & 0.06 & -0.01 \\
\hline & 8 & 0.06 & 0.07 & -0.16 & 0.07 & 0.16 \\
\hline & 9 & 0.02 & 0.02 & 0.04 & 0.02 & -0.04 \\
\hline & 10 & 0.13 & 0.14 & -0.44 & 0.15 & 0.45 \\
\hline \multirow{12}{*}{ cn_5 } & 1 & 0.13 & 0.14 & -0.44 & 0.14 & 0.44 \\
\hline & 2 & 0.02 & 0.02 & 0.04 & 0.02 & -0.04 \\
\hline & 3 & 0.06 & 0.07 & -0.16 & 0.07 & 0.16 \\
\hline & 4 & 0.06 & 0.06 & 0.02 & 0.06 & -0.01 \\
\hline & 5 & 0.07 & 0.07 & -0.04 & 0.07 & 0.04 \\
\hline & 6 & 0.06 & 0.06 & 0.01 & 0.06 & 0.00 \\
\hline & 7 & 0.06 & 0.06 & -0.01 & 0.06 & 0.00 \\
\hline & 8 & 0.07 & 0.07 & 0.04 & 0.07 & 0.04 \\
\hline & 9 & 0.06 & 0.06 & -0.02 & 0.06 & -0.01 \\
\hline & 10 & 0.06 & 0.07 & 0.16 & 0.07 & 0.16 \\
\hline & 11 & 0.02 & 0.02 & -0.04 & 0.02 & -0.04 \\
\hline & 12 & 0.13 & 0.14 & 0.44 & 0.14 & 0.44 \\
\hline
\end{tabular}


Table S25. Computed Mulliken Charge (Spin) densities of the selected carbons in figure S4 for the CS, OSS and T (OSS and T) states of R=BiPh Chichibabin systems, at OPBE/cc-pVTZ level of theory.

\begin{tabular}{|c|c|c|c|c|c|c|}
\hline \multirow{2}{*}{ Structure } & \multirow{2}{*}{ Atom } & \multirow{2}{*}{$\begin{array}{c}\text { CS } \\
\text { Charge }\end{array}$} & \multicolumn{2}{|c|}{ OSS } & \multicolumn{2}{|c|}{$\mathbf{T}$} \\
\hline & & & Charge & Spin & Charge & Spin \\
\hline \multirow{4}{*}{ biph_1 } & 1 & 0.03 & 0.03 & 0.00 & 0.06 & 0.50 \\
\hline & 2 & 0.05 & 0.05 & 0.00 & 0.02 & 0.01 \\
\hline & 3 & 0.05 & 0.05 & 0.00 & 0.02 & 0.01 \\
\hline & 4 & 0.03 & 0.03 & 0.00 & 0.06 & 0.50 \\
\hline \multirow{6}{*}{ biph_2 } & 1 & 0.04 & 0.05 & -0.43 & 0.06 & 0.48 \\
\hline & 2 & 0.03 & 0.02 & 0.06 & 0.02 & -0.03 \\
\hline & 3 & 0.07 & 0.06 & -0.08 & 0.06 & 0.05 \\
\hline & 4 & 0.07 & 0.06 & 0.08 & 0.06 & 0.05 \\
\hline & 5 & 0.03 & 0.02 & -0.06 & 0.02 & -0.03 \\
\hline & 6 & 0.04 & 0.05 & 0.43 & 0.06 & 0.48 \\
\hline \multirow{8}{*}{ biph_3 } & 1 & 0.04 & 0.06 & -0.47 & 0.06 & 0.48 \\
\hline & 2 & 0.03 & 0.02 & 0.05 & 0.02 & -0.04 \\
\hline & 3 & 0.06 & 0.06 & -0.07 & 0.06 & 0.07 \\
\hline & 4 & 0.06 & 0.06 & 0.02 & 0.06 & 0.00 \\
\hline & 5 & 0.06 & 0.06 & -0.02 & 0.06 & 0.00 \\
\hline & 6 & 0.06 & 0.06 & 0.07 & 0.06 & 0.06 \\
\hline & 7 & 0.03 & 0.02 & -0.04 & 0.02 & -0.04 \\
\hline & 8 & 0.04 & 0.06 & 0.47 & 0.06 & 0.48 \\
\hline \multirow{10}{*}{ biph_4 } & 1 & 0.05 & 0.06 & 0.48 & 0.06 & 0.48 \\
\hline & 2 & 0.02 & 0.02 & -0.04 & 0.02 & -0.04 \\
\hline & 3 & 0.06 & 0.06 & 0.07 & 0.06 & 0.07 \\
\hline & 4 & 0.06 & 0.06 & -0.01 & 0.06 & -0.01 \\
\hline & 5 & 0.06 & 0.06 & 0.01 & 0.06 & 0.01 \\
\hline & 6 & 0.06 & 0.06 & -0.01 & 0.06 & 0.01 \\
\hline & 7 & 0.06 & 0.06 & 0.01 & 0.06 & -0.01 \\
\hline & 8 & 0.06 & 0.06 & -0.07 & 0.06 & 0.07 \\
\hline & 9 & 0.02 & 0.02 & 0.04 & 0.02 & -0.04 \\
\hline & 10 & 0.05 & 0.06 & -0.48 & 0.06 & 0.48 \\
\hline \multirow{12}{*}{ biph_5 } & 1 & 0.05 & 0.06 & 0.48 & 0.06 & 0.48 \\
\hline & 2 & 0.02 & 0.02 & -0.04 & 0.02 & -0.04 \\
\hline & 3 & 0.06 & 0.06 & 0.07 & 0.06 & 0.07 \\
\hline & 4 & 0.06 & 0.06 & -0.01 & 0.06 & -0.01 \\
\hline & 5 & 0.06 & 0.06 & 0.01 & 0.06 & 0.01 \\
\hline & 6 & 0.06 & 0.06 & 0.00 & 0.06 & 0.00 \\
\hline & 7 & 0.06 & 0.06 & 0.00 & 0.06 & 0.00 \\
\hline & 8 & 0.06 & 0.06 & -0.01 & 0.06 & 0.01 \\
\hline & 9 & 0.06 & 0.06 & 0.01 & 0.06 & -0.01 \\
\hline & 10 & 0.06 & 0.06 & -0.07 & 0.06 & 0.07 \\
\hline & 11 & 0.02 & 0.02 & 0.04 & 0.02 & -0.04 \\
\hline & 12 & 0.05 & 0.06 & -0.48 & 0.06 & 0.48 \\
\hline
\end{tabular}


Table S26. Computed Mulliken Charge (Spin) densities of the selected carbons in figure S4 for the CS, OSS and T (OSS and T) states of $\mathrm{R}=\mathrm{Ph}$ Chichibabin systems, at OPBE/cc-pVTZ level of theory.

\begin{tabular}{|c|c|c|c|c|c|c|}
\hline \multirow{2}{*}{ Structure } & \multirow{2}{*}{ Atom } & \multirow{2}{*}{$\begin{array}{c}\text { CS } \\
\text { Charge }\end{array}$} & \multicolumn{2}{|c|}{ OSS } & \multicolumn{2}{|c|}{$\mathbf{T}$} \\
\hline & & & Charge & Spin & Charge & Spin \\
\hline \multirow{4}{*}{ ph_1 } & 1 & 0.09 & 0.09 & 0.00 & 0.10 & 0.56 \\
\hline & 2 & -0.01 & -0.01 & 0.00 & -0.01 & 0.02 \\
\hline & 3 & -0.01 & -0.01 & 0.00 & -0.01 & 0.02 \\
\hline & 4 & 0.09 & 0.09 & 0.00 & -0.11 & 0.04 \\
\hline \multirow{6}{*}{ ph_2 } & 1 & 0.10 & 0.10 & 0.00 & 0.11 & 0.55 \\
\hline & 2 & -0.01 & -0.01 & 0.00 & 0.00 & -0.05 \\
\hline & 3 & 0.06 & 0.06 & 0.00 & 0.06 & 0.09 \\
\hline & 4 & 0.06 & 0.06 & 0.00 & 0.06 & 0.09 \\
\hline & 5 & -0.01 & -0.01 & 0.00 & 0.00 & -0.05 \\
\hline & 6 & 0.10 & 0.10 & 0.00 & 0.11 & 0.55 \\
\hline \multirow{8}{*}{ ph_3 } & 1 & 0.10 & 0.11 & 0.52 & 0.11 & 0.54 \\
\hline & 2 & 0.00 & 0.00 & -0.07 & 0.00 & -0.06 \\
\hline & 3 & 0.06 & 0.06 & 0.12 & 0.06 & 0.11 \\
\hline & 4 & 0.06 & 0.06 & -0.04 & 0.06 & 0.01 \\
\hline & 5 & 0.06 & 0.06 & 0.04 & 0.06 & 0.01 \\
\hline & 6 & 0.06 & 0.06 & -0.12 & 0.06 & 0.11 \\
\hline & 7 & 0.00 & 0.00 & 0.07 & 0.00 & -0.06 \\
\hline & 8 & 0.10 & 0.11 & -0.52 & 0.11 & 0.54 \\
\hline \multirow{10}{*}{ ph_4 } & 1 & 0.10 & 0.11 & -0.54 & 0.11 & 0.54 \\
\hline & 2 & 0.00 & 0.00 & 0.06 & 0.00 & -0.06 \\
\hline & 3 & 0.05 & 0.06 & -0.11 & 0.06 & 0.11 \\
\hline & 4 & 0.06 & 0.06 & 0.02 & 0.06 & -0.01 \\
\hline & 5 & 0.06 & 0.06 & -0.03 & 0.06 & 0.02 \\
\hline & 6 & 0.06 & 0.06 & 0.03 & 0.06 & 0.02 \\
\hline & 7 & 0.06 & 0.06 & -0.02 & 0.06 & -0.01 \\
\hline & 8 & 0.05 & 0.06 & 0.11 & 0.06 & 0.11 \\
\hline & 9 & 0.00 & 0.00 & -0.06 & 0.00 & -0.06 \\
\hline & 10 & 0.10 & 0.11 & 0.54 & 0.11 & 0.54 \\
\hline \multirow{12}{*}{ ph_5 } & 1 & 0.10 & 0.11 & -0.54 & 0.11 & 0.54 \\
\hline & 2 & 0.00 & 0.00 & 0.06 & 0.00 & -0.06 \\
\hline & 3 & 0.05 & 0.05 & -0.12 & 0.05 & 0.12 \\
\hline & 4 & 0.06 & 0.06 & 0.02 & 0.06 & -0.02 \\
\hline & 5 & 0.06 & 0.06 & -0.02 & 0.06 & 0.02 \\
\hline & 6 & 0.06 & 0.06 & 0.01 & 0.06 & 0.00 \\
\hline & 7 & 0.06 & 0.06 & -0.01 & 0.06 & 0.00 \\
\hline & 8 & 0.06 & 0.06 & 0.02 & 0.06 & 0.02 \\
\hline & 9 & 0.06 & 0.06 & -0.02 & 0.06 & -0.02 \\
\hline & 10 & 0.05 & 0.05 & 0.12 & 0.05 & 0.12 \\
\hline & 11 & 0.00 & 0.00 & -0.06 & 0.00 & -0.06 \\
\hline & 12 & 0.10 & 0.11 & 0.54 & 0.11 & 0.54 \\
\hline
\end{tabular}


Table S27. Computed Mulliken Charge (Spin) densities of the selected carbons in figure S4 for the CS, OSS and $\mathrm{T}$ (OSS and T) states of $\mathrm{R}=p$-FPh Chichibabin systems, at OPBE/cc-pVTZ level of theory.

\begin{tabular}{|c|c|c|c|c|c|c|}
\hline \multirow{2}{*}{ Structure } & \multirow{2}{*}{ Atom } & \multirow{2}{*}{$\begin{array}{c}\text { CS } \\
\text { Charge }\end{array}$} & \multicolumn{2}{|c|}{ OSS } & \multicolumn{2}{|c|}{$\mathbf{T}$} \\
\hline & & & Charge & Spin & Charge & Spin \\
\hline \multirow{4}{*}{ p_fph_1 } & 1 & 0.08 & 0.08 & 0.00 & 0.10 & 0.56 \\
\hline & 2 & 0.00 & 0.00 & 0.00 & -0.01 & 0.02 \\
\hline & 3 & 0.00 & 0.00 & 0.00 & -0.01 & 0.02 \\
\hline & 4 & 0.08 & 0.08 & 0.00 & 0.10 & 0.56 \\
\hline \multirow{6}{*}{ p_fph_2 } & 1 & 0.09 & 0.09 & 0.00 & 0.10 & 0.54 \\
\hline & 2 & 0.00 & 0.00 & 0.00 & 0.00 & -0.04 \\
\hline & 3 & 0.05 & 0.05 & 0.00 & 0.05 & 0.09 \\
\hline & 4 & 0.05 & 0.05 & 0.00 & 0.05 & 0.09 \\
\hline & 5 & 0.00 & 0.00 & 0.00 & 0.00 & -0.04 \\
\hline & 6 & 0.09 & 0.09 & 0.00 & 0.10 & 0.54 \\
\hline \multirow{8}{*}{ p_fph_3 } & 1 & 0.09 & 0.10 & 0.51 & 0.10 & 0.54 \\
\hline & 2 & 0.00 & 0.00 & -0.06 & 0.00 & -0.06 \\
\hline & 3 & 0.06 & 0.05 & 0.12 & 0.06 & 0.11 \\
\hline & 4 & 0.06 & 0.06 & -0.04 & 0.06 & 0.01 \\
\hline & 5 & 0.06 & 0.06 & 0.04 & 0.06 & 0.01 \\
\hline & 6 & 0.06 & 0.05 & -0.12 & 0.06 & 0.11 \\
\hline & 7 & 0.00 & 0.00 & 0.06 & 0.00 & -0.06 \\
\hline & 8 & 0.09 & 0.10 & -0.51 & 0.10 & 0.54 \\
\hline \multirow{10}{*}{ p_fph_4 } & 1 & 0.09 & 0.10 & -0.53 & 0.10 & 0.53 \\
\hline & 2 & 0.00 & 0.00 & 0.06 & 0.00 & -0.06 \\
\hline & 3 & 0.05 & 0.06 & -0.11 & 0.06 & 0.11 \\
\hline & 4 & 0.06 & 0.06 & 0.02 & 0.06 & -0.01 \\
\hline & 5 & 0.06 & 0.06 & -0.03 & 0.06 & 0.02 \\
\hline & 6 & 0.06 & 0.06 & 0.03 & 0.06 & 0.02 \\
\hline & 7 & 0.06 & 0.06 & -0.02 & 0.06 & -0.01 \\
\hline & 8 & 0.05 & 0.06 & 0.11 & 0.06 & 0.11 \\
\hline & 9 & 0.00 & 0.00 & -0.06 & 0.00 & -0.06 \\
\hline & 10 & 0.09 & 0.10 & 0.53 & 0.10 & 0.53 \\
\hline \multirow{12}{*}{ p_fph_5 } & 1 & 0.10 & 0.10 & 0.53 & 0.10 & 0.53 \\
\hline & 2 & 0.00 & 0.00 & -0.06 & 0.00 & -0.06 \\
\hline & 3 & 0.05 & 0.05 & 0.12 & 0.05 & 0.12 \\
\hline & 4 & 0.06 & 0.06 & -0.02 & 0.06 & -0.02 \\
\hline & 5 & 0.06 & 0.06 & 0.02 & 0.06 & 0.02 \\
\hline & 6 & 0.06 & 0.06 & -0.01 & 0.06 & 0.00 \\
\hline & 7 & 0.06 & 0.06 & 0.01 & 0.06 & 0.00 \\
\hline & 8 & 0.06 & 0.06 & -0.02 & 0.06 & 0.02 \\
\hline & 9 & 0.06 & 0.06 & 0.02 & 0.06 & -0.02 \\
\hline & 10 & 0.05 & 0.05 & -0.12 & 0.05 & 0.12 \\
\hline & 11 & 0.00 & 0.00 & 0.06 & 0.00 & -0.06 \\
\hline & 12 & 0.10 & 0.10 & -0.53 & 0.10 & 0.53 \\
\hline
\end{tabular}


Table S28. Computed Mulliken Charge (Spin) densities of the selected carbons in figure S4 for the CS, OSS and $\mathrm{T}(\mathrm{OSS}$ and $\mathrm{T})$ states of $\mathrm{R}=\mathrm{p}-\mathrm{NO}_{2} \mathrm{Ph}$ Chichibabin systems, at OPBE/cc-pVTZ level of theory.

\begin{tabular}{|c|c|c|c|c|c|c|}
\hline \multirow{2}{*}{ Structure } & \multirow{2}{*}{ Atom } & \multirow{2}{*}{$\begin{array}{c}\text { CS } \\
\text { Charge }\end{array}$} & \multicolumn{2}{|c|}{ OSS } & \multicolumn{2}{|c|}{$\mathbf{T}$} \\
\hline & & & Charge & Spin & Charge & Spin \\
\hline \multirow{4}{*}{ p_noph_1 } & 1 & 0.11 & 0.11 & 0.00 & 0.13 & 0.51 \\
\hline & 2 & 0.00 & 0.00 & 0.00 & -0.02 & 0.02 \\
\hline & 3 & 0.00 & 0.00 & 0.00 & -0.02 & 0.02 \\
\hline & 4 & 0.11 & 0.11 & 0.00 & 0.13 & 0.51 \\
\hline \multirow{6}{*}{ p_noph_2 } & 1 & 0.12 & 0.12 & 0.00 & 0.12 & 0.50 \\
\hline & 2 & 0.00 & 0.00 & 0.00 & 0.00 & -0.04 \\
\hline & 3 & 0.06 & 0.06 & 0.00 & 0.06 & 0.07 \\
\hline & 4 & 0.06 & 0.06 & 0.00 & 0.06 & 0.07 \\
\hline & 5 & 0.00 & 0.00 & 0.00 & 0.00 & -0.04 \\
\hline & 6 & 0.12 & 0.12 & 0.00 & 0.12 & 0.50 \\
\hline \multirow{8}{*}{ p_noph_3 } & 1 & 0.12 & 0.13 & 0.47 & 0.13 & 0.49 \\
\hline & 2 & 0.00 & -0.01 & -0.05 & -0.01 & -0.05 \\
\hline & 3 & 0.06 & 0.06 & 0.09 & 0.06 & 0.09 \\
\hline & 4 & 0.06 & 0.06 & -0.03 & 0.06 & 0.00 \\
\hline & 5 & 0.06 & 0.06 & 0.03 & 0.06 & 0.00 \\
\hline & 6 & 0.06 & 0.06 & -0.09 & 0.06 & 0.09 \\
\hline & 7 & 0.00 & -0.01 & 0.05 & -0.01 & -0.05 \\
\hline & 8 & 0.12 & 0.13 & -0.47 & 0.13 & 0.49 \\
\hline \multirow{10}{*}{ p_noph_4 } & 1 & 0.12 & 0.13 & 0.48 & 0.13 & 0.49 \\
\hline & 2 & 0.00 & -0.01 & -0.05 & -0.01 & -0.05 \\
\hline & 3 & 0.06 & 0.06 & 0.09 & 0.06 & 0.09 \\
\hline & 4 & 0.06 & 0.06 & -0.01 & 0.06 & -0.01 \\
\hline & 5 & 0.06 & 0.06 & 0.02 & 0.06 & 0.02 \\
\hline & 6 & 0.06 & 0.06 & -0.02 & 0.06 & 0.02 \\
\hline & 7 & 0.06 & 0.06 & 0.01 & 0.06 & -0.01 \\
\hline & 8 & 0.06 & 0.06 & -0.09 & 0.06 & 0.09 \\
\hline & 9 & 0.00 & -0.01 & 0.05 & -0.01 & -0.05 \\
\hline & 10 & 0.12 & 0.13 & -0.48 & 0.13 & 0.49 \\
\hline \multirow{12}{*}{ p_noph_5 } & 1 & 0.12 & 0.13 & 0.48 & 0.13 & 0.48 \\
\hline & 2 & 0.00 & -0.01 & -0.05 & -0.01 & -0.05 \\
\hline & 3 & 0.06 & 0.06 & 0.10 & 0.06 & 0.10 \\
\hline & 4 & 0.06 & 0.06 & -0.01 & 0.06 & -0.01 \\
\hline & 5 & 0.06 & 0.06 & 0.02 & 0.06 & 0.02 \\
\hline & 6 & 0.06 & 0.06 & -0.01 & 0.06 & 0.00 \\
\hline & 7 & 0.06 & 0.06 & 0.01 & 0.06 & 0.00 \\
\hline & 8 & 0.06 & 0.06 & -0.02 & 0.06 & 0.02 \\
\hline & 9 & 0.06 & 0.06 & 0.01 & 0.06 & -0.01 \\
\hline & 10 & 0.06 & 0.06 & -0.10 & 0.06 & 0.10 \\
\hline & 11 & 0.00 & -0.01 & 0.05 & -0.01 & -0.05 \\
\hline & 12 & 0.12 & 0.13 & -0.48 & 0.13 & 0.48 \\
\hline
\end{tabular}


Table S29. Computed Mulliken Charge (Spin) densities of the selected carbons in figure S4 for the CS, OSS and T (OSS and T) states of R=FPh Chichibabin systems, at OPBE/cc-pVTZ level of theory.

\begin{tabular}{|c|c|c|c|c|c|c|}
\hline \multirow{2}{*}{ Structure } & \multirow{2}{*}{ Atom } & \multirow{2}{*}{$\begin{array}{c}\text { CS } \\
\text { Charge }\end{array}$} & \multicolumn{2}{|c|}{ OSS } & \multicolumn{2}{|c|}{$\mathbf{T}$} \\
\hline & & & Charge & Spin & Charge & Spin \\
\hline \multirow{4}{*}{ fph_1 } & 1 & 0.15 & 0.15 & 0.00 & 0.14 & 0.63 \\
\hline & 2 & 0.01 & 0.01 & 0.00 & 0.03 & 0.02 \\
\hline & 3 & 0.01 & 0.01 & 0.00 & 0.03 & 0.02 \\
\hline & 4 & 0.15 & 0.15 & 0.00 & 0.14 & 0.63 \\
\hline \multirow{6}{*}{ fph_2 } & 1 & 0.15 & 0.15 & 0.00 & 0.14 & 0.61 \\
\hline & 2 & 0.01 & 0.01 & 0.00 & 0.03 & -0.06 \\
\hline & 3 & 0.07 & 0.07 & 0.00 & 0.06 & 0.12 \\
\hline & 4 & 0.07 & 0.07 & 0.00 & 0.06 & 0.12 \\
\hline & 5 & 0.01 & 0.01 & 0.00 & 0.03 & -0.06 \\
\hline & 6 & 0.15 & 0.15 & 0.00 & 0.14 & 0.61 \\
\hline \multirow{8}{*}{ fph_3 } & 1 & 0.14 & 0.14 & -0.56 & 0.14 & 0.60 \\
\hline & 2 & 0.02 & 0.03 & 0.08 & 0.03 & -0.07 \\
\hline & 3 & 0.07 & 0.06 & -0.15 & 0.06 & 0.15 \\
\hline & 4 & 0.07 & 0.06 & 0.05 & 0.06 & 0.01 \\
\hline & 5 & 0.07 & 0.06 & -0.05 & 0.06 & 0.01 \\
\hline & 6 & 0.07 & 0.06 & 0.15 & 0.06 & 0.15 \\
\hline & 7 & 0.02 & 0.03 & -0.08 & 0.03 & -0.07 \\
\hline & 8 & 0.14 & 0.14 & 0.56 & 0.14 & 0.60 \\
\hline \multirow{10}{*}{ fph_4 } & 1 & 0.13 & 0.14 & -0.59 & 0.14 & 0.59 \\
\hline & 2 & 0.03 & 0.03 & 0.08 & 0.03 & -0.08 \\
\hline & 3 & 0.06 & 0.06 & -0.15 & 0.06 & 0.15 \\
\hline & 4 & 0.06 & 0.06 & 0.03 & 0.06 & -0.02 \\
\hline & 5 & 0.06 & 0.06 & -0.03 & 0.06 & 0.03 \\
\hline & 6 & 0.06 & 0.06 & 0.03 & 0.06 & 0.03 \\
\hline & 7 & 0.06 & 0.06 & -0.03 & 0.06 & -0.02 \\
\hline & 8 & 0.06 & 0.06 & 0.15 & 0.06 & 0.15 \\
\hline & 9 & 0.03 & 0.03 & -0.08 & 0.03 & -0.08 \\
\hline & 10 & 0.13 & 0.14 & 0.59 & 0.14 & 0.59 \\
\hline \multirow{12}{*}{ fph_5 } & 1 & 0.13 & 0.14 & -0.59 & 0.14 & 0.59 \\
\hline & 2 & 0.02 & 0.02 & 0.08 & 0.02 & -0.08 \\
\hline & 3 & 0.06 & 0.06 & -0.15 & 0.06 & 0.15 \\
\hline & 4 & 0.06 & 0.06 & 0.02 & 0.06 & -0.02 \\
\hline & 5 & 0.06 & 0.06 & -0.03 & 0.06 & 0.03 \\
\hline & 6 & 0.06 & 0.06 & 0.01 & 0.06 & 0.00 \\
\hline & 7 & 0.06 & 0.06 & -0.01 & 0.06 & 0.00 \\
\hline & 8 & 0.06 & 0.06 & 0.03 & 0.06 & 0.03 \\
\hline & 9 & 0.06 & 0.06 & -0.02 & 0.06 & -0.02 \\
\hline & 10 & 0.06 & 0.06 & 0.15 & 0.06 & 0.15 \\
\hline & 11 & 0.02 & 0.02 & -0.08 & 0.02 & -0.08 \\
\hline & 12 & 0.13 & 0.14 & 0.59 & 0.14 & 0.59 \\
\hline
\end{tabular}


Table S30. Computed Mulliken Charge (Spin) densities of the selected carbons in figure S4 for the CS, OSS and $\mathrm{T}$ (OSS and T) states of $\mathrm{R}=\mathrm{NO}_{2} \mathrm{Ph}$ Chichibabin systems, at OPBE/cc-pVTZ level of theory.

\begin{tabular}{|c|c|c|c|c|c|c|}
\hline \multirow{2}{*}{ Structure } & \multirow{2}{*}{ Atom } & \multirow{2}{*}{$\begin{array}{c}\text { CS } \\
\text { Charge }\end{array}$} & \multicolumn{2}{|c|}{ OSS } & \multicolumn{2}{|c|}{$\mathbf{T}$} \\
\hline & & & Charge & Spin & Charge & Spin \\
\hline \multirow{4}{*}{ noph_1 } & 1 & 0.01 & 0.01 & 0.00 & 0.08 & 0.63 \\
\hline & 2 & -0.03 & -0.03 & 0.00 & -0.10 & 0.02 \\
\hline & 3 & -0.03 & -0.03 & 0.00 & -0.10 & 0.02 \\
\hline & 4 & 0.01 & 0.01 & 0.00 & 0.08 & 0.63 \\
\hline \multirow{6}{*}{ noph_2 } & 1 & 0.05 & 0.05 & -0.27 & 0.07 & 0.58 \\
\hline & 2 & -0.05 & -0.06 & 0.04 & -0.11 & -0.03 \\
\hline & 3 & 0.06 & 0.06 & -0.09 & 0.06 & 0.10 \\
\hline & 4 & 0.06 & 0.06 & 0.09 & 0.06 & 0.10 \\
\hline & 5 & -0.05 & -0.06 & -0.04 & -0.11 & -0.03 \\
\hline & 6 & 0.05 & 0.05 & 0.27 & 0.07 & 0.58 \\
\hline \multirow{8}{*}{ noph_3 } & 1 & 0.04 & 0.06 & 0.48 & 0.07 & 0.51 \\
\hline & 2 & -0.07 & -0.11 & -0.03 & -0.12 & -0.03 \\
\hline & 3 & 0.07 & 0.07 & 0.11 & 0.07 & 0.10 \\
\hline & 4 & 0.07 & 0.06 & -0.04 & 0.06 & 0.01 \\
\hline & 5 & 0.07 & 0.06 & 0.04 & 0.06 & 0.01 \\
\hline & 6 & 0.07 & 0.07 & -0.11 & 0.07 & 0.10 \\
\hline & 7 & -0.07 & -0.11 & 0.03 & -0.12 & -0.03 \\
\hline & 8 & 0.04 & 0.06 & -0.48 & 0.07 & 0.51 \\
\hline \multirow{10}{*}{ noph_4 } & 1 & 0.05 & 0.06 & -0.48 & 0.06 & 0.49 \\
\hline & 2 & -0.08 & -0.12 & 0.02 & -0.12 & -0.02 \\
\hline & 3 & 0.07 & 0.07 & -0.11 & 0.07 & 0.10 \\
\hline & 4 & 0.06 & 0.01 & 0.05 & 0.05 & 0.00 \\
\hline & 5 & 0.07 & 0.07 & -0.03 & 0.07 & 0.03 \\
\hline & 6 & 0.07 & 0.07 & 0.03 & 0.07 & 0.03 \\
\hline & 7 & 0.06 & -0.01 & 0.05 & 0.05 & 0.00 \\
\hline & 8 & 0.07 & 0.07 & 0.11 & 0.07 & 0.10 \\
\hline & 9 & -0.08 & -0.12 & -0.02 & -0.12 & -0.02 \\
\hline & 10 & 0.05 & 0.06 & 0.48 & 0.06 & 0.49 \\
\hline \multirow{12}{*}{ noph_5 } & 1 & 0.05 & 0.06 & -0.50 & 0.06 & 0.50 \\
\hline & 2 & -0.09 & -0.10 & 0.03 & -0.10 & -0.02 \\
\hline & 3 & 0.06 & 0.07 & -0.12 & 0.07 & 0.12 \\
\hline & 4 & 0.06 & 0.05 & 0.01 & 0.05 & -0.01 \\
\hline & 5 & 0.07 & 0.07 & -0.04 & 0.07 & 0.03 \\
\hline & 6 & 0.06 & 0.06 & 0.01 & 0.06 & 0.01 \\
\hline & 7 & 0.06 & 0.06 & -0.01 & 0.06 & 0.01 \\
\hline & 8 & 0.07 & 0.07 & 0.04 & 0.07 & 0.03 \\
\hline & 9 & 0.06 & 0.05 & -0.01 & 0.05 & -0.01 \\
\hline & 10 & 0.06 & 0.07 & 0.12 & 0.07 & 0.12 \\
\hline & 11 & -0.09 & -0.10 & -0.03 & -0.10 & -0.02 \\
\hline & 12 & 0.05 & 0.06 & 0.50 & 0.06 & 0.50 \\
\hline
\end{tabular}




\section{III.4 Aromaticity:}

Table S31. HOMA Aromaticity results (in a.u.) of the studied Chichibabin systems in the CS, OSS and T states. For the type-A', A, B, and C rings (depicted in the scheme below).

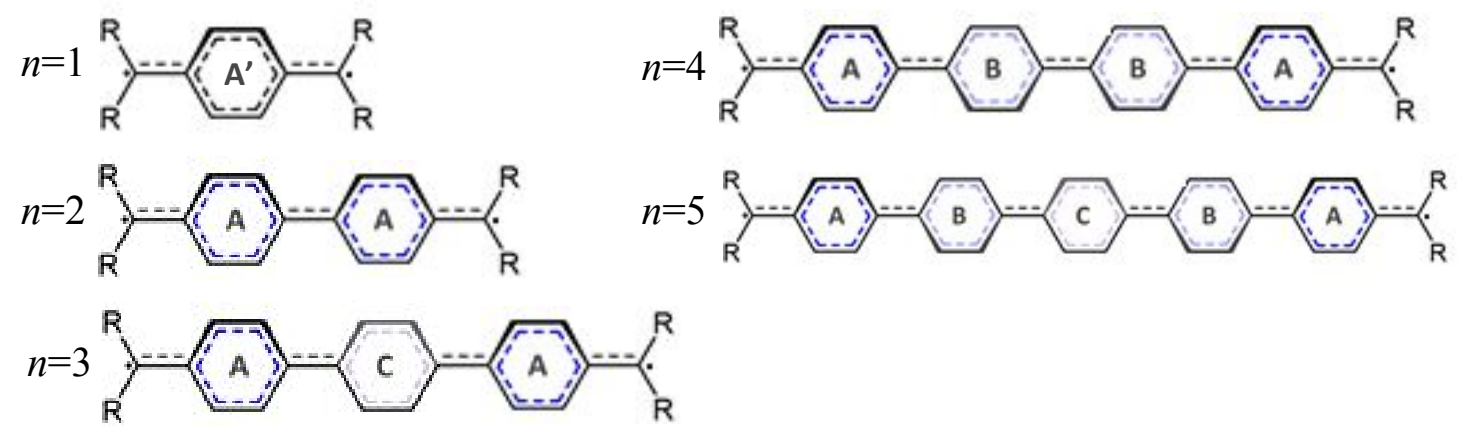

\begin{tabular}{|c|c|c|c|c|c|c|c|c|c|c|c|c|}
\hline \multirow{2}{*}{$n$} & \multirow{2}{*}{ State } & \multicolumn{11}{|c|}{ Type-A' and A Ring } \\
\hline & & H & $\mathrm{OH}$ & NH2 & $\mathrm{CN}$ & $\mathrm{COH}$ & $\mathrm{Ph}$ & FPh & NOPh & p_FPh & p_NO2Ph & $\mathrm{BiPh}$ \\
\hline \multirow[t]{4}{*}{1} & $\mathrm{CS}$ & 0.200 & 0.327 & 0.375 & 0.545 & 0.393 & 0.445 & 0.413 & 0.451 & 0.454 & 0.495 & 0.703 \\
\hline & OsS & 0.200 & 0.327 & 0.375 & 0.545 & 0.393 & 0.445 & 0.413 & 0.451 & 0.454 & 0.495 & 0.703 \\
\hline & $\mathrm{T}$ & 0.893 & 0.862 & 0.853 & 0.923 & 0.737 & 0.918 & 0.920 & 0.904 & 0.916 & 0.920 & 0.936 \\
\hline & $\mathrm{CS}$ & 0.468 & 0.535 & 0.570 & 0.695 & 0.652 & 0.668 & 0.633 & 0.707 & 0.674 & 0.721 & 0.829 \\
\hline \multirow[t]{3}{*}{2} & OsS & 0.468 & 0.535 & 0.566 & 0.695 & 0.652 & 0.668 & 0.633 & 0.752 & 0.674 & 0.721 & 0.914 \\
\hline & $\mathrm{T}$ & 0.857 & 0.850 & 0.828 & 0.908 & 0.914 & 0.918 & 0.910 & 0.910 & 0.917 & 0.926 & 0.940 \\
\hline & $\mathrm{CS}$ & 0.576 & 0.636 & 0.663 & 0.780 & 0.735 & 0.785 & 0.747 & 0.807 & 0.789 & 0.840 & 0.877 \\
\hline \multirow[t]{3}{*}{3} & OSS & 0.773 & 0.725 & 0.665 & 0.861 & 0.829 & 0.896 & 0.872 & 0.892 & 0.895 & 0.913 & 0.932 \\
\hline & $\mathrm{T}$ & 0.833 & 0.826 & 0.805 & 0.893 & 0.868 & 0.912 & 0.897 & 0.907 & 0.911 & 0.923 & 0.936 \\
\hline & $\mathrm{CS}$ & 0.648 & 0.700 & 0.719 & 0.824 & 0.814 & 0.848 & 0.818 & 0.843 & 0.849 & 0.884 & 0.905 \\
\hline \multirow[t]{3}{*}{4} & OSS & 0.817 & 0.775 & 0.719 & 0.881 & 0.869 & 0.908 & 0.889 & 0.897 & 0.907 & 0.918 & 0.935 \\
\hline & $\mathrm{T}$ & 0.824 & 0.791 & 0.798 & 0.886 & 0.875 & 0.910 & 0.893 & 0.900 & 0.909 & 0.920 & 0.936 \\
\hline & $\mathrm{CS}$ & 0.697 & 0.740 & 0.752 & 0.845 & 0.844 & 0.866 & 0.841 & 0.847 & 0.866 & 0.889 & 0.915 \\
\hline \multirow[t]{2}{*}{5} & OsS & 0.822 & 0.814 & 0.793 & 0.883 & 0.871 & 0.905 & 0.887 & 0.880 & 0.904 & 0.915 & 0.936 \\
\hline & $\mathrm{T}$ & 0.823 & 0.815 & 0.796 & 0.884 & 0.872 & 0.905 & 0.888 & 0.881 & 0.904 & 0.915 & 0.936 \\
\hline
\end{tabular}

\begin{tabular}{|c|c|c|c|c|c|c|c|c|c|c|c|c|}
\hline \multirow{2}{*}{$n$} & \multirow{2}{*}{ State } & \multicolumn{11}{|c|}{ Type-B Ring } \\
\hline & & H & $\mathrm{OH}$ & NH2 & $\mathrm{CN}$ & $\mathrm{COH}$ & $\mathrm{Ph}$ & FPh & NOPh & p_FPh & p_NO2Ph & $\mathrm{BiPh}$ \\
\hline \multirow{3}{*}{4} & $\mathrm{CS}$ & 0.858 & 0.858 & 0.852 & 0.898 & 0.908 & 0.923 & 0.910 & 0.910 & 0.924 & 0.937 & 0.947 \\
\hline & OSS & 0.946 & 0.924 & 0.852 & 0.944 & 0.948 & 0.954 & 0.951 & 0.947 & 0.954 & 0.956 & 0.958 \\
\hline & $\mathrm{T}$ & 0.950 & 0.939 & 0.937 & 0.949 & 0.951 & 0.955 & 0.954 & 0.950 & 0.955 & 0.957 & 0.959 \\
\hline \multirow{3}{*}{5} & $\mathrm{CS}$ & 0.911 & 0.907 & 0.899 & 0.926 & 0.930 & 0.941 & 0.934 & 0.925 & 0.941 & 0.947 & 0.953 \\
\hline & OSS & 0.951 & 0.945 & 0.935 & 0.948 & 0.950 & 0.955 & 0.953 & 0.946 & 0.955 & 0.956 & 0.959 \\
\hline & $\mathrm{T}$ & 0.950 & 0.946 & 0.938 & 0.949 & 0.951 & 0.955 & 0.954 & 0.947 & 0.955 & 0.956 & 0.959 \\
\hline
\end{tabular}

\begin{tabular}{ccccccccccccc}
\hline \multirow{2}{n}{$n$} & \multirow{2}{*}{ State } & \multicolumn{10}{c}{ Type-C Ring } \\
& & $\mathbf{H}$ & $\mathbf{O H}$ & $\mathbf{N H 2}$ & $\mathbf{C N}$ & $\mathbf{C O H}$ & $\mathbf{P h}$ & $\mathbf{F P h}$ & NOPh & p_FPh & p_NO2Ph & BiPh \\
\hline & CS & 0.755 & 0.766 & 0.770 & 0.844 & 0.834 & 0.862 & 0.836 & 0.867 & 0.864 & 0.896 & 0.917 \\
$\mathbf{3}$ & OSS & 0.910 & 0.854 & 0.773 & 0.919 & 0.917 & 0.944 & 0.936 & 0.938 & 0.943 & 0.950 & 0.954 \\
& T & 0.948 & 0.941 & 0.932 & 0.948 & 0.946 & 0.953 & 0.952 & 0.952 & 0.953 & 0.956 & 0.957 \\
& CS & 0.929 & 0.925 & 0.918 & 0.936 & 0.944 & 0.951 & 0.947 & 0.939 & 0.951 & 0.954 & 0.955 \\
$\mathbf{5}$ & OSS & 0.957 & 0.954 & 0.923 & 0.956 & 0.958 & 0.960 & 0.959 & 0.956 & 0.960 & 0.960 & 0.960 \\
& T & 0.959 & 0.956 & 0.952 & 0.957 & 0.959 & 0.960 & 0.960 & 0.957 & 0.960 & 0.960 & 0.960 \\
\hline
\end{tabular}


Table S32. PDI Aromaticity results (in a.u.) of the studied Chichibabin systems in the CS, OSS and T states. For the type-A', A, B, and C rings (depicted in the scheme of Table S31).

\begin{tabular}{|c|c|c|c|c|c|c|c|c|c|c|c|c|}
\hline \multirow{2}{*}{$n$} & \multirow{2}{*}{ State } & \multicolumn{11}{|c|}{ Type-A' and A Ring } \\
\hline & & H & $\mathrm{OH}$ & NH2 & $\mathrm{CN}$ & $\mathrm{COH}$ & $\mathrm{Ph}$ & FPh & NOPh & p_FPh & p_NO2Ph & $\mathrm{BiPh}$ \\
\hline \multirow{3}{*}{1} & $\mathrm{CS}$ & 0.031 & 0.033 & 0.036 & 0.041 & 0.038 & 0.040 & 0.037 & 0.040 & 0.041 & 0.043 & 0.054 \\
\hline & OSS & 0.031 & 0.033 & 0.036 & 0.041 & 0.038 & 0.040 & 0.037 & 0.040 & 0.041 & 0.043 & 0.054 \\
\hline & $\mathrm{T}$ & 0.084 & 0.079 & 0.079 & 0.081 & 0.063 & 0.084 & 0.081 & 0.079 & 0.083 & 0.084 & 0.088 \\
\hline \multirow{3}{*}{2} & $\mathrm{CS}$ & 0.040 & 0.042 & 0.044 & 0.051 & 0.051 & 0.052 & 0.048 & 0.054 & 0.052 & 0.056 & 0.066 \\
\hline & OSS & 0.040 & 0.042 & 0.044 & 0.051 & 0.051 & 0.052 & 0.048 & 0.058 & 0.052 & 0.056 & 0.081 \\
\hline & $\mathrm{T}$ & 0.075 & 0.073 & 0.072 & 0.078 & 0.080 & 0.083 & 0.079 & 0.079 & 0.082 & 0.084 & 0.087 \\
\hline \multirow{3}{*}{3} & $\mathrm{CS}$ & 0.046 & 0.048 & 0.050 & 0.059 & 0.058 & 0.062 & 0.056 & 0.062 & 0.062 & 0.068 & 0.074 \\
\hline & OSS & 0.063 & 0.056 & 0.051 & 0.069 & 0.069 & 0.078 & 0.072 & 0.075 & 0.078 & 0.081 & 0.085 \\
\hline & $\mathrm{T}$ & 0.070 & 0.068 & 0.068 & 0.075 & 0.075 & 0.081 & 0.077 & 0.078 & 0.081 & 0.083 & 0.086 \\
\hline \multirow{3}{*}{4} & $\mathrm{CS}$ & 0.051 & 0.053 & 0.054 & 0.063 & 0.065 & 0.070 & 0.064 & 0.067 & 0.070 & 0.075 & 0.079 \\
\hline & OSS & 0.068 & 0.062 & 0.063 & 0.073 & 0.075 & 0.080 & 0.075 & 0.076 & 0.080 & 0.082 & 0.086 \\
\hline & $\mathrm{T}$ & 0.069 & 0.065 & 0.066 & 0.074 & 0.075 & 0.081 & 0.076 & 0.076 & 0.081 & 0.082 & 0.086 \\
\hline \multirow{3}{*}{5} & $\mathrm{CS}$ & 0.055 & 0.056 & 0.058 & 0.066 & 0.069 & 0.073 & 0.068 & 0.068 & 0.073 & 0.076 & 0.081 \\
\hline & OSS & 0.069 & 0.067 & 0.065 & 0.073 & 0.075 & 0.080 & 0.075 & 0.074 & 0.080 & 0.081 & 0.086 \\
\hline & $\mathrm{T}$ & 0.069 & 0.067 & 0.066 & 0.073 & 0.075 & 0.080 & 0.075 & 0.074 & 0.080 & 0.081 & 0.086 \\
\hline \multirow{2}{*}{$n$} & \multirow{2}{*}{ State } & \multicolumn{11}{|c|}{ Type-B Ring } \\
\hline & & $\mathbf{H}$ & $\mathrm{OH}$ & NH2 & $\mathrm{CN}$ & $\mathrm{COH}$ & $\mathrm{Ph}$ & FPh & NOPh & p_FPh & p_NO2Ph & $\mathrm{BiPh}$ \\
\hline \multirow{3}{*}{4} & $\mathrm{CS}$ & 0.070 & 0.070 & 0.069 & 0.076 & 0.078 & 0.081 & 0.078 & 0.078 & 0.082 & 0.084 & 0.087 \\
\hline & OSS & 0.087 & 0.082 & 0.081 & 0.086 & 0.087 & 0.090 & 0.089 & 0.087 & 0.090 & 0.090 & 0.091 \\
\hline & $\mathrm{T}$ & 0.089 & 0.086 & 0.086 & 0.088 & 0.088 & 0.090 & 0.090 & 0.088 & 0.090 & 0.090 & 0.091 \\
\hline \multirow{3}{*}{5} & $\mathrm{CS}$ & 0.079 & 0.078 & 0.077 & 0.081 & 0.082 & 0.086 & 0.084 & 0.081 & 0.086 & 0.087 & 0.089 \\
\hline & OSS & 0.089 & 0.087 & 0.085 & 0.087 & 0.088 & 0.090 & 0.089 & 0.087 & 0.090 & 0.090 & 0.091 \\
\hline & $\mathrm{T}$ & 0.089 & 0.088 & 0.086 & 0.088 & 0.088 & 0.090 & 0.089 & 0.087 & 0.090 & 0.090 & 0.091 \\
\hline \multirow{2}{*}{$n$} & \multirow{2}{*}{ State } & \multicolumn{11}{|c|}{ Type-C Ring } \\
\hline & & $\mathbf{H}$ & OH & NH2 & $\mathrm{CN}$ & $\mathrm{COH}$ & Ph & FPh & NOPh & p_FPh & p_NO2Ph & BiPh \\
\hline \multirow{3}{*}{3} & $\mathrm{CS}$ & 0.058 & 0.059 & 0.059 & 0.067 & 0.066 & 0.071 & 0.067 & 0.070 & 0.071 & 0.076 & 0.080 \\
\hline & OSS & 0.079 & 0.070 & 0.060 & 0.080 & 0.079 & 0.087 & 0.084 & 0.084 & 0.086 & 0.088 & 0.090 \\
\hline & $\mathrm{T}$ & 0.088 & 0.087 & 0.085 & 0.087 & 0.087 & 0.090 & 0.089 & 0.088 & 0.090 & 0.090 & 0.090 \\
\hline \multirow{3}{*}{5} & $\mathrm{CS}$ & 0.083 & 0.082 & 0.081 & 0.084 & 0.086 & 0.089 & 0.087 & 0.084 & 0.089 & 0.089 & 0.090 \\
\hline & OSS & 0.091 & 0.090 & 0.088 & 0.090 & 0.091 & 0.091 & 0.091 & 0.090 & 0.091 & 0.091 & 0.092 \\
\hline & $\mathrm{T}$ & 0.091 & 0.090 & 0.089 & 0.090 & 0.091 & 0.092 & 0.091 & 0.090 & 0.092 & 0.092 & 0.092 \\
\hline
\end{tabular}


Table S33. FLU Aromaticity results (in a.u.) of the studied Chichibabin systems in the CS, OSS and T states. For the type-A', A, B and C rings (depicted in the scheme of Table S31).

\begin{tabular}{|c|c|c|c|c|c|c|c|c|c|c|c|c|c|}
\hline \multirow{2}{*}{$n$} & \multirow{2}{*}{ State } & \multicolumn{12}{|c|}{ Type-A' and A Ring } \\
\hline & & H & OH & NH2 & $\mathrm{CN}$ & $\mathrm{COH}$ & $\mathbf{P h}$ & FPh & & OPh p & p_FPh & p_NO2Ph & BiPh \\
\hline \multirow{3}{*}{1} & $\mathrm{CS}$ & 0.038 & 0.034 & $0.029 \quad 0$ & 0.025 & 0.028 & 0.026 & 0.030 & & .027 & 0.026 & 0.024 & 0.015 \\
\hline & OSS & 0.038 & 0.034 & $0.029 \quad 0$ & 0.025 & 0.028 & 0.026 & 0.030 & & .027 & 0.026 & 0.024 & 0.015 \\
\hline & $\mathrm{T}$ & 0.005 & 0.007 & 0.006 & 0.005 & 0.013 & 0.004 & 0.005 & & .006 & 0.004 & 0.004 & 0.003 \\
\hline \multirow{3}{*}{2} & $\mathrm{CS}$ & 0.026 & 0.024 & 0.021 & 0.017 & 0.017 & 0.017 & 0.019 & & .016 & 0.016 & 0.014 & 0.009 \\
\hline & OSS & 0.026 & 0.024 & $0.021 \quad 0$ & 0.017 & 0.017 & 0.017 & 0.019 & & .013 & 0.016 & 0.014 & 0.005 \\
\hline & $\mathrm{T}$ & 0.007 & 0.008 & $0.008 \quad 0$ & 0.006 & 0.005 & 0.004 & 0.005 & & .005 & 0.004 & 0.004 & 0.003 \\
\hline \multirow{3}{*}{3} & $\mathrm{CS}$ & 0.021 & 0.019 & 0.017 & 0.013 & 0.013 & 0.011 & 0.014 & & .011 & 0.011 & 0.009 & 0.006 \\
\hline & OSS & 0.012 & 0.014 & $0.016 \quad 0$ & 0.008 & 0.009 & 0.005 & 0.007 & & .007 & 0.005 & 0.005 & 0.003 \\
\hline & $\mathrm{T}$ & 0.008 & 0.009 & 0.009 & 0.006 & 0.007 & 0.005 & 0.006 & & .006 & 0.005 & 0.004 & 0.003 \\
\hline \multirow{3}{*}{4} & $\mathrm{CS}$ & 0.018 & 0.016 & 0.014 & 0.010 & 0.010 & 0.008 & 0.010 & & .009 & 0.008 & 0.006 & 0.005 \\
\hline & OSS & 0.009 & 0.011 & 0.011 & 0.007 & 0.007 & 0.005 & 0.007 & & .006 & 0.005 & 0.004 & 0.003 \\
\hline & $\mathrm{T}$ & 0.009 & 0.010 & $0.010 \quad 0$ & 0.007 & 0.006 & 0.005 & 0.006 & & .006 & 0.005 & 0.004 & 0.003 \\
\hline \multirow{3}{*}{5} & $\mathrm{CS}$ & 0.015 & 0.014 & 0.012 & 0.009 & 0.008 & 0.007 & 0.009 & & .009 & 0.007 & 0.006 & 0.004 \\
\hline & OSS & 0.009 & 0.010 & 0.010 & 0.007 & 0.007 & 0.005 & 0.007 & & .007 & 0.005 & 0.005 & 0.003 \\
\hline & $\mathrm{T}$ & 0.009 & 0.010 & $0.010 \quad 0$ & 0.007 & 0.007 & 0.005 & 0.006 & & .007 & 0.005 & 0.005 & 0.003 \\
\hline \multirow{2}{*}{$n$} & \multirow{2}{*}{ State } & \multicolumn{12}{|c|}{ Type-B Ring } \\
\hline & & H & $\mathrm{OH}$ & NH2 & $\mathrm{CN}$ & $\mathrm{COH}$ & $\mathrm{Ph}$ & $\mathbf{F P h}$ & & $\mathrm{OPh}$ & p_FPh & p_NO2Ph & $\mathrm{BiPh}$ \\
\hline \multirow{3}{*}{4} & $\mathrm{CS}$ & 0.008 & 0.007 & $0.008 \quad 0$ & 0.006 & 0.005 & 0.004 & 0.005 & & .005 & 0.004 & 0.003 & 0.003 \\
\hline & OSS & 0.003 & 0.004 & 0.004 & 0.003 & 0.003 & 0.002 & 0.003 & & .003 & 0.002 & 0.002 & 0.002 \\
\hline & $\mathrm{T}$ & 0.003 & 0.003 & 0.003 & 0.003 & 0.003 & 0.002 & 0.002 & & .003 & 0.002 & 0.002 & 0.002 \\
\hline \multirow{3}{*}{5} & $\mathrm{CS}$ & 0.005 & 0.005 & 0.005 & 0.004 & 0.004 & 0.003 & 0.004 & & .004 & 0.003 & 0.003 & 0.003 \\
\hline & OSS & 0.003 & 0.003 & 0.003 & 0.003 & 0.003 & 0.002 & 0.003 & & .003 & 0.002 & 0.002 & 0.002 \\
\hline & $\mathrm{T}$ & 0.003 & 0.003 & 0.003 & 0.003 & 0.003 & 0.002 & 0.003 & & .003 & 0.002 & 0.002 & 0.002 \\
\hline \multirow{2}{*}{$n$} & \multirow{2}{*}{ State } & \multicolumn{12}{|c|}{ Type-C Ring } \\
\hline & & & $\mathbf{H}$ & $\mathrm{OH}$ & NH2 & $\mathrm{CN}$ & $\mathrm{COH}$ & $\mathrm{Ph}$ & FPh & NOPh & h_FPh & h p_NO2Ph & $\mathrm{BiPh}$ \\
\hline \multirow{3}{*}{3} & $\mathrm{CS}$ & & 0.013 & 0.012 & 0.012 & 0.009 & 0.009 & 0.007 & 0.009 & 0.007 & 0.007 & 0.006 & 0.005 \\
\hline & OSS & & 0.005 & 0.008 & 0.011 & 0.005 & 0.005 & 0.003 & 0.004 & 0.004 & 0.003 & 0.003 & 0.002 \\
\hline & $\mathrm{T}$ & & 0.003 & 0.003 & 0.003 & 0.003 & 0.003 & 0.002 & 0.003 & 0.003 & 0.002 & 0.002 & 0.002 \\
\hline \multirow{3}{*}{5} & $\mathrm{CS}$ & & 0.004 & 0.004 & 0.004 & 0.004 & 0.003 & 0.003 & 0.003 & 0.004 & 0.003 & 0.003 & 0.002 \\
\hline & OSS & & 0.002 & 0.002 & 0.003 & 0.002 & 0.002 & 0.002 & 0.002 & 0.002 & 0.002 & 0.002 & 0.002 \\
\hline & $\mathrm{T}$ & & 0.002 & 0.002 & 0.002 & 0.002 & 0.002 & 0.002 & 0.002 & 0.002 & 0.002 & 0.002 & 0.002 \\
\hline
\end{tabular}


Table S34. I $_{\text {ring }}$ Aromaticity results (in a.u.) of the studied Chichibabin systems in the CS, OSS and T states. For the type-A', A, B and C rings (depicted in the scheme of Table S31).

\begin{tabular}{|c|c|c|c|c|c|c|c|c|c|c|c|c|c|}
\hline \multirow{2}{*}{$n$} & \multirow{2}{*}{ State } & \multicolumn{12}{|c|}{ Type-A' and A Ring } \\
\hline & & H & $\mathrm{OH}$ & NH2 & $\mathrm{CN}$ & $\mathrm{COH}$ & $\mathbf{H}$ & $h$ & FPh & NOPh & p_FPh & p_NO2Ph & $\mathrm{BiPh}$ \\
\hline \multirow{3}{*}{1} & $\mathrm{CS}$ & 0.011 & 0.012 & 0.013 & $\begin{array}{ll}3 & 0.017\end{array}$ & 0.01 & 0.0 & 16 & 0.014 & 0.016 & 0.016 & 0.017 & 0.023 \\
\hline & OSS & 0.011 & 0.012 & 0.013 & 0.017 & 0.01 & 0.0 & 16 & 0.014 & 0.016 & 0.016 & 0.017 & 0.023 \\
\hline & $\mathrm{T}$ & 0.030 & 0.028 & 0.028 & 0.032 & 0.02 & $\begin{array}{ll}26 & 0.0\end{array}$ & 340. & 0.032 & 0.030 & 0.034 & 0.034 & 0.037 \\
\hline \multirow{3}{*}{2} & $\mathrm{CS}$ & 0.016 & 0.017 & 0.017 & 0.022 & 0.02 & 0.0 & 22 & 0.020 & 0.022 & 0.022 & 0.024 & 0.029 \\
\hline & OSS & 0.016 & 0.017 & 0.017 & 0.022 & 0.02 & 0.0 & 22 & 0.020 & 0.024 & 0.022 & 0.024 & 0.034 \\
\hline & $\mathrm{T}$ & 0.029 & 0.028 & 0.028 & 0.032 & 0.03 & 0.0 & 34 & 0.033 & 0.032 & 0.034 & 0.035 & 0.037 \\
\hline \multirow{3}{*}{3} & $\mathrm{CS}$ & 0.019 & 0.019 & 0.020 & 0.025 & 0.02 & 0.0 & 26 & 0.024 & 0.026 & 0.026 & 0.029 & 0.032 \\
\hline & OSS & 0.025 & 0.022 & 0.020 & 0.029 & 0.02 & 0.0 & 32 & .030 & 0.031 & 0.032 & 0.034 & 0.036 \\
\hline & $\mathrm{T}$ & 0.028 & 0.027 & 0.026 & 0.031 & 0.03 & 0.0 & 340. & .032 & 0.032 & 0.034 & 0.035 & 0.037 \\
\hline \multirow{3}{*}{4} & $\mathrm{CS}$ & 0.020 & 0.021 & 0.022 & 0.027 & 0.02 & 0.0 & 29 & .027 & 0.028 & 0.029 & 0.032 & 0.034 \\
\hline & OSS & 0.027 & 0.024 & 0.025 & 0.031 & 0.03 & 0.0 & 33 & .031 & 0.031 & 0.033 & 0.034 & 0.037 \\
\hline & $\mathrm{T}$ & 0.027 & 0.025 & 0.026 & 0.031 & 0.03 & 0.0 & 34 & .031 & 0.031 & 0.033 & 0.034 & 0.037 \\
\hline \multirow{3}{*}{5} & $\mathrm{CS}$ & 0.022 & 0.023 & 0.023 & 0.028 & 0.03 & 0.0 & 30 & .028 & 0.028 & 0.030 & 0.032 & 0.035 \\
\hline & OSS & 0.027 & 0.026 & 0.026 & 0.031 & 0.03 & 0.0 & 33 & .031 & 0.030 & 0.033 & 0.034 & 0.037 \\
\hline & $\mathrm{T}$ & 0.027 & 0.026 & 0.026 & 0.031 & 0.03 & 0.0 & 33 & .031 & 0.030 & 0.033 & 0.034 & 0.037 \\
\hline \multirow{2}{*}{$n$} & \multirow{2}{*}{ State } & \multicolumn{12}{|c|}{ Type-B Ring } \\
\hline & & \multicolumn{2}{|r|}{$\mathrm{H}$} & $\mathrm{OH}$ & NH2 & $\mathrm{CN}$ & $\mathrm{COH}$ & $\mathrm{Ph}$ & FPh & NOPh & p_FPh & p_NO2Ph & $\mathrm{BiPh}$ \\
\hline \multirow{3}{*}{4} & $\mathrm{CS}$ & \multicolumn{2}{|c|}{0.030} & 0.030 & 0.030 & 0.033 & 0.034 & 0.035 & \multirow{2}{*}{$\begin{array}{ll}5 & 0.034 \\
8 & 0.038\end{array}$} & 0.034 & 0.035 & 0.036 & 0.038 \\
\hline & OSS & \multicolumn{2}{|c|}{0.037} & 0.035 & 0.034 & 0.037 & 0.038 & 0.038 & & 0.037 & 0.038 & 0.039 & 0.039 \\
\hline & $\mathrm{T}$ & \multicolumn{2}{|c|}{0.038} & 0.037 & 0.036 & 0.038 & 0.038 & 0.039 & $\begin{array}{ll}9 & 0.038\end{array}$ & 0.038 & 0.039 & 0.039 & 0.039 \\
\hline \multirow{3}{*}{5} & $\mathrm{CS}$ & \multicolumn{2}{|c|}{0.034} & 0.033 & 0.033 & 0.035 & 0.036 & 0.037 & 0.036 & 0.035 & 0.037 & 0.038 & 0.038 \\
\hline & OSS & & 0.038 & 0.037 & 0.036 & 0.038 & 0.038 & 0.039 & 0.038 & 0.037 & 0.039 & 0.039 & 0.039 \\
\hline & $\mathrm{T}$ & & 0.038 & 0.037 & 0.036 & 0.038 & 0.038 & 0.039 & 0.038 & 0.038 & 0.039 & 0.039 & 0.039 \\
\hline$n$ & State & & & & & & Тур & e-C Ri & ing & & & & \\
\hline$n$ & Netet & $\mathbf{H}$ & $\mathrm{OH}$ & NH2 & $\mathrm{CN}$ & $\mathrm{COH}$ & $\mathbf{P h}$ & FPh & NO & $\mathrm{Ph}$ & D_FPh & p_NO2Ph & $\mathrm{BiPh}$ \\
\hline & CS & 0.025 & 0.025 & 0.025 & 0.030 & 0.029 & 0.031 & 0.029 & 0.0 & 031 & 0.031 & 0.033 & 0.035 \\
\hline 3 & OSS & 0.034 & 0.030 & 0.025 & 0.035 & 0.034 & 0.037 & 0.036 & 0.0 & 036 & 0.037 & 0.038 & 0.039 \\
\hline & $\mathrm{T}$ & 0.038 & 0.037 & 0.036 & 0.038 & 0.037 & 0.038 & 0.038 & 0.0 & 038 & 0.038 & 0.039 & 0.039 \\
\hline & CS & 0.036 & 0.035 & 0.034 & 0.036 & 0.037 & 0.038 & 0.038 & 0.0 & 037 & 0.038 & 0.039 & 0.039 \\
\hline 5 & OSS & 0.039 & 0.039 & 0.038 & 0.039 & 0.039 & 0.039 & 0.039 & 0.0 & 039 & 0.039 & 0.039 & 0.039 \\
\hline & $\mathrm{T}$ & 0.039 & 0.039 & 0.038 & 0.039 & 0.039 & 0.039 & 0.039 & 0.0 & 039 & 0.039 & 0.039 & 0.039 \\
\hline
\end{tabular}


Table S35. MCI Aromaticity results (in a.u.) of the studied Chichibabin systems in the CS, OSS and T states. For the type-A' A, B and C rings (depicted in the scheme of Table S31).

\begin{tabular}{|c|c|c|c|c|c|c|c|c|c|c|c|c|}
\hline \multirow{2}{*}{$n$} & \multirow{2}{*}{ State } & \multicolumn{11}{|c|}{ Type-A' and A Ring } \\
\hline & & H & $\mathrm{OH}$ & NH2 & $\mathrm{CN}$ & $\mathrm{COH}$ & $\mathrm{Ph}$ & FPh & NOPh & p_FPh & p_NO2Ph & $\mathrm{BiPh}$ \\
\hline \multirow{4}{*}{1} & $\mathrm{CS}$ & 0.013 & 0.014 & 0.015 & 0.021 & 0.018 & 0.019 & 0.017 & 0.019 & 0.019 & 0.021 & 0.030 \\
\hline & OSS & 0.013 & 0.014 & 0.015 & 0.021 & 0.018 & 0.019 & 0.017 & 0.019 & 0.019 & 0.021 & 0.030 \\
\hline & $\mathrm{T}$ & 0.043 & 0.039 & 0.039 & 0.044 & 0.035 & 0.048 & 0.046 & 0.043 & 0.048 & 0.049 & 0.054 \\
\hline & $\mathrm{CS}$ & 0.019 & 0.020 & 0.021 & 0.028 & 0.028 & 0.028 & 0.025 & 0.029 & 0.028 & 0.031 & 0.039 \\
\hline \multirow[t]{2}{*}{2} & OSS & 0.019 & 0.020 & 0.021 & 0.028 & 0.028 & 0.028 & 0.025 & 0.031 & 0.028 & 0.031 & 0.049 \\
\hline & $\mathrm{T}$ & 0.041 & 0.039 & 0.038 & 0.045 & 0.049 & 0.049 & 0.046 & 0.045 & 0.049 & 0.050 & 0.054 \\
\hline \multirow{3}{*}{3} & $\mathrm{CS}$ & 0.023 & 0.024 & 0.025 & 0.033 & 0.032 & 0.035 & 0.031 & 0.035 & 0.035 & 0.039 & 0.044 \\
\hline & OSS & 0.033 & 0.029 & 0.026 & 0.040 & 0.039 & 0.046 & 0.041 & 0.043 & 0.045 & 0.048 & 0.052 \\
\hline & $\mathrm{T}$ & 0.038 & 0.036 & 0.036 & 0.043 & 0.043 & 0.048 & 0.044 & 0.045 & 0.048 & 0.049 & 0.053 \\
\hline \multirow{3}{*}{4} & $\mathrm{CS}$ & 0.026 & 0.027 & 0.028 & 0.037 & 0.038 & 0.040 & 0.036 & 0.038 & 0.040 & 0.044 & 0.048 \\
\hline & OSS & 0.037 & 0.032 & 0.034 & 0.042 & 0.043 & 0.047 & 0.043 & 0.043 & 0.047 & 0.049 & 0.053 \\
\hline & $\mathrm{T}$ & 0.037 & 0.034 & 0.035 & 0.042 & 0.044 & 0.048 & 0.044 & 0.044 & 0.047 & 0.049 & 0.053 \\
\hline \multirow{3}{*}{5} & $\mathrm{CS}$ & 0.029 & 0.030 & 0.030 & 0.038 & 0.041 & 0.042 & 0.038 & 0.038 & 0.042 & 0.045 & 0.049 \\
\hline & OSS & 0.037 & 0.035 & 0.035 & 0.042 & 0.043 & 0.047 & 0.043 & 0.042 & 0.047 & 0.048 & 0.053 \\
\hline & $\mathrm{T}$ & 0.037 & 0.035 & 0.035 & 0.042 & 0.043 & 0.047 & 0.043 & 0.042 & 0.047 & 0.048 & 0.053 \\
\hline
\end{tabular}

\begin{tabular}{|c|c|c|c|c|c|c|c|c|c|c|c|c|}
\hline \multirow{2}{*}{$n$} & \multirow{2}{*}{ State } & \multicolumn{11}{|c|}{ Type-B Ring } \\
\hline & & H & $\mathrm{OH}$ & NH2 & $\mathrm{CN}$ & $\mathrm{COH}$ & $\mathrm{Ph}$ & FPh & NOPh & p_FPh & p_NO2Ph & $\mathrm{BiPh}$ \\
\hline \multirow{3}{*}{4} & $\mathrm{CS}$ & 0.042 & 0.041 & 0.040 & 0.046 & 0.047 & 0.050 & 0.048 & 0.048 & 0.050 & 0.052 & 0.054 \\
\hline & OS & 0.054 & 0.050 & 0.049 & 0.054 & 0.054 & 0.056 & 0.055 & 0.054 & 0.056 & 0.056 & 0.057 \\
\hline & $\mathrm{T}$ & 0.055 & 0.053 & 0.052 & 0.055 & 0.055 & 0.056 & 0.056 & 0.055 & 0.056 & 0.057 & 0.057 \\
\hline \multirow{3}{*}{5} & $\mathrm{CS}$ & 0.048 & 0.047 & 0.046 & 0.050 & 0.051 & 0.053 & 0.052 & 0.050 & 0.053 & 0.054 & 0.056 \\
\hline & OS & 0.055 & 0.054 & 0.052 & 0.054 & 0.055 & 0.056 & 0.056 & 0.054 & 0.056 & 0.056 & 0.057 \\
\hline & $\mathrm{T}$ & 0.055 & 0.054 & 0.052 & 0.055 & 0.055 & 0.056 & 0.056 & 0.054 & 0.056 & 0.056 & 0.057 \\
\hline
\end{tabular}

\begin{tabular}{ccccccccccccc}
\hline \multirow{2}{*}{$n$} & \multirow{2}{*}{ State } & $\mathbf{1 0}$ & $\mathbf{1 0}$ & $\mathbf{1 0}$ \\
& & $\mathbf{H}$ & OH & NH2 & CN & COH & Ph & FPh & NOPh & p_FPh & p_NO2Ph & BiPh \\
\hline \multirow{3}{*}{3} & CS & 0.033 & 0.033 & 0.033 & 0.040 & 0.039 & 0.042 & 0.039 & 0.042 & 0.042 & 0.046 & 0.049 \\
& OS & 0.048 & 0.041 & 0.034 & 0.049 & 0.049 & 0.054 & 0.052 & 0.052 & 0.053 & 0.055 & 0.056 \\
& T & 0.055 & 0.053 & 0.051 & 0.054 & 0.054 & 0.056 & 0.055 & 0.055 & 0.056 & 0.056 & 0.057 \\
& CS & 0.051 & 0.050 & 0.049 & 0.052 & 0.053 & 0.055 & 0.054 & 0.052 & 0.055 & 0.056 & 0.056 \\
$\mathbf{5}$ & OS & 0.057 & 0.056 & 0.055 & 0.056 & 0.057 & 0.057 & 0.057 & 0.056 & 0.057 & 0.057 & 0.058 \\
& T & 0.057 & 0.056 & 0.056 & 0.057 & 0.057 & 0.057 & 0.057 & 0.057 & 0.057 & 0.058 & 0.058 \\
\hline
\end{tabular}




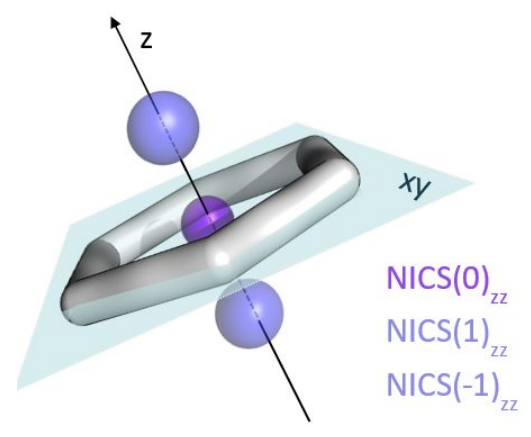

Figure S5. Representation of the ghost atom positions (purple spheres) for the calculation of $\operatorname{NICS}(0)_{z z}, \operatorname{NICS}(1)_{z z}$ and $\operatorname{NICS}(-1)_{\mathrm{zz}}$.

Table S36. $\operatorname{NICS}_{\mathrm{ZZ}}(0,1$ and -1) Aromaticity results (in ppm) of the studied Chichibabin systems in the CS, OSS and T states. For the type-A' and A rings (depicted in the scheme of Table S31).

\begin{tabular}{|c|c|c|c|c|c|c|c|c|c|}
\hline \multirow{2}{*}{ Structure } & \multicolumn{3}{|c|}{$\mathrm{CS}$} & \multicolumn{3}{|c|}{ OSS } & \multicolumn{3}{|c|}{$\mathrm{T}$} \\
\hline & $\operatorname{NICS}(0)$ & NICS(1) & NICS(-1) & $\operatorname{NICS}(0)$ & $\operatorname{NICS}(1)$ & NICS(-1) & $\operatorname{NICS}(0)$ & NICS(1) & NICS(-1) \\
\hline h_1 & 18.1 & -1.9 & -1.9 & 18.1 & -1.9 & -1.9 & -3.3 & -19.6 & -19.6 \\
\hline h_2 & 16.0 & -4.2 & -4.2 & 16.0 & -4.2 & -4.2 & -0.3 & -16.9 & -16.9 \\
\hline h_3 & 13.6 & -5.7 & -5.7 & 5.7 & -12.3 & -12.3 & 1.8 & -15.2 & -15.2 \\
\hline h_4 & 12.1 & -7.3 & -7.3 & 3.2 & -14.2 & -14.2 & 2.7 & -14.6 & -14.6 \\
\hline h_5 & 10.2 & -8.6 & -8.6 & 2.8 & -14.5 & -14.5 & 2.8 & -14.5 & -14.5 \\
\hline oh_1 & 22.9 & 1.4 & 1.4 & 22.9 & 1.4 & 1.4 & 6.0 & -11.9 & -13.2 \\
\hline oh_2 & 18.1 & -2.9 & -2.9 & 18.1 & -2.9 & -2.9 & 4.5 & -13.4 & -13.4 \\
\hline oh_3 & 11.1 & -5.1 & -5.1 & 12.1 & -7.5 & -7.5 & 6.5 & -11.8 & -11.8 \\
\hline oh_4 & 13.8 & -6.2 & -6.2 & 8.1 & -10.6 & -10.6 & 7.2 & -11.2 & -11.2 \\
\hline oh 5 & 12.7 & -7.0 & -7.0 & 7.6 & -11.0 & -11.0 & 7.5 & -11.1 & -11.1 \\
\hline nh_1 & 28.0 & 5.4 & 5.4 & 28.0 & 5.4 & 5.4 & 6.5 & -12.3 & -12.4 \\
\hline nh_2 & 20.3 & -0.9 & -0.9 & 20.3 & -0.9 & -0.9 & 10.5 & -8.7 & -8.7 \\
\hline nh_3 & 18.5 & -2.6 & -2.6 & 18.4 & -2.7 & -2.7 & 12.2 & -7.4 & -7.4 \\
\hline$n h_{-}^{-} 4$ & 17.3 & -3.6 & -3.6 & 13.8 & -6.3 & -6.3 & 13.0 & -6.9 & -6.9 \\
\hline nh_5 & 16.6 & -4.2 & -4.2 & 13.3 & -6.7 & -6.7 & 13.2 & -6.8 & -6.8 \\
\hline coh_1 & 16.0 & -3.7 & -3.7 & 16.0 & -3.7 & -3.7 & 2.7 & -13.0 & -13.0 \\
\hline coh_2 & 10.0 & -8.9 & -8.9 & 10.0 & -8.9 & -8.9 & 0.8 & -16.0 & -16.0 \\
\hline coh_3 & 8.4 & -10.3 & -10.3 & 1.6 & -15.6 & -15.6 & -0.5 & -17.0 & -17.0 \\
\hline coh_4 & 5.7 & -12.3 & -12.3 & 0.2 & -16.6 & -16.6 & -0.2 & -16.9 & -16.9 \\
\hline $\operatorname{coh}^{-} 5$ & 4.2 & -13.4 & -13.4 & 0.2 & -16.6 & -16.6 & 0.1 & -16.7 & -16.7 \\
\hline cn_1 & 14.3 & -5.8 & -5.8 & 14.3 & -5.8 & -5.8 & -1.9 & -19.1 & -19.1 \\
\hline cn_2 & 10.6 & -8.9 & -8.9 & 10.6 & -8.9 & -8.9 & -1.4 & -18.2 & -18.2 \\
\hline cn_3 & 7.5 & -11.4 & -11.4 & 2.6 & -15.2 & -15.2 & 0.0 & -17.1 & -17.1 \\
\hline cn_4 & 5.7 & -12.7 & -12.7 & 1.4 & -16.1 & -16.1 & 0.9 & -16.4 & -16.4 \\
\hline cn_5 & 4.2 & -13.8 & -13.8 & 1.0 & -16.3 & -16.3 & 0.8 & -16.4 & -16.4 \\
\hline biph_1 & 8.9 & -9.1 & -9.1 & 8.9 & -9.1 & -9.1 & -8.3 & -22.8 & -22.8 \\
\hline biph_2 & 3.9 & -13.7 & -13.7 & -3.9 & -19.7 & -19.7 & -7.3 & -22.2 & -22.2 \\
\hline biph_3 & 0.2 & -16.5 & -16.5 & -6.0 & -21.3 & -21.3 & -6.4 & -21.6 & -21.7 \\
\hline biph_4 & -2.1 & -18.2 & -18.2 & -5.8 & -21.1 & -21.1 & -5.9 & -21.2 & -21.2 \\
\hline biph 5 & -4.0 & -19.7 & -19.7 & -6.6 & -21.7 & -21.8 & -6.6 & -21.8 & -21.8 \\
\hline ph_1 & 15.4 & -4.2 & -4.2 & 15.4 & -4.2 & -4.2 & -5.5 & -20.6 & -20.6 \\
\hline ph_2 & 11.6 & -7.8 & -7.8 & 11.6 & -7.8 & -7.8 & -4.0 & -19.8 & -19.8 \\
\hline ph_3 & 7.2 & -11.3 & -11.3 & -1.5 & -18.0 & -18.0 & -3.3 & -19.3 & -19.3 \\
\hline ph_4 & 3.4 & -14.2 & -14.2 & -2.6 & -18.8 & -18.8 & -2.8 & -19.0 & -19.0 \\
\hline ph_5 & 1.7 & -15.4 & -15.4 & -2.4 & -18.6 & -18.6 & -2.5 & -18.6 & -18.6 \\
\hline p_fph_1 & 15.5 & -4.1 & -4.1 & 15.5 & -4.1 & -4.1 & -4.9 & -20.2 & -20.2 \\
\hline p_fph_2 & 11.5 & -7.8 & -7.8 & 11.5 & -7.8 & -7.8 & -3.6 & -19.6 & -19.6 \\
\hline p_fph_3 & 7.2 & -11.3 & -11.3 & -1.2 & -17.7 & -17.7 & -3.1 & -19.1 & -19.1 \\
\hline p_fph_4 & 3.5 & -14.1 & -14.1 & -2.4 & -18.6 & -18.6 & -2.6 & -18.7 & -18.7 \\
\hline p_fph 5 & 1.9 & -15.2 & -15.2 & -2.2 & -18.4 & -18.4 & -2.2 & -18.4 & -18.4 \\
\hline p_noph_1 & 13.4 & -5.8 & -5.8 & 13.4 & -5.8 & -5.8 & -6.8 & -21.6 & -21.6 \\
\hline p_noph_2 & 8.8 & -10.0 & -10.0 & 8.8 & -10.0 & -10.0 & -5.8 & -21.2 & -21.2 \\
\hline p_noph_3 & 2.7 & -14.7 & -14.7 & -3.4 & -19.4 & -19.4 & -5.4 & -20.9 & -20.9 \\
\hline p_noph_4 & -0.9 & -17.4 & -17.4 & -4.7 & -20.3 & -20.3 & -4.9 & -20.5 & -20.5 \\
\hline p_noph 5 & -1.5 & -17.9 & -17.9 & -4.3 & -20.0 & -20.0 & -4.3 & -20.1 & -20.1 \\
\hline fph_1 & 16.3 & -3.5 & -3.5 & 16.3 & -3.5 & -3.5 & -5.0 & -20.2 & -20.2 \\
\hline fph_2 & 12.7 & -6.9 & -6.9 & 12.7 & -6.9 & -6.9 & -2.9 & -18.9 & -19.0 \\
\hline fph_3 & 8.9 & -9.9 & -9.9 & 0.8 & -16.2 & -16.2 & -1.6 & -17.9 & -17.9 \\
\hline fph_4 & 5.2 & -12.7 & -12.7 & -0.6 & -17.2 & -17.2 & -1.1 & -17.5 & -17.5 \\
\hline fph_5 & 3.5 & -13.9 & -13.9 & -0.6 & -17.1 & -17.1 & -0.6 & -17.2 & -17.2 \\
\hline
\end{tabular}




\begin{tabular}{lccc|ccc|ccc} 
noph_1 & 11.8 & -6.7 & -6.7 & 11.8 & -6.7 & -6.7 & -6.4 & -21.6 & -21.6 \\
noph_2 & 8.1 & -10.4 & -10.4 & 6.1 & -12.0 & -12.0 & -5.0 & -20.6 & -20.6 \\
noph_3 & 3.6 & -13.9 & -13.9 & -3.5 & -19.4 & -19.4 & -5.2 & -20.6 & -20.6 \\
noph_4 & 1.5 & -15.5 & -15.5 & -3.7 & -19.5 & -19.5 & -4.0 & -19.7 & -19.7 \\
noph_5 & 1.3 & -15.6 & -15.6 & -1.7 & -18.0 & -18.0 & -1.8 & -18.1 & -18.1 \\
\hline
\end{tabular}

Table S37. NICS $\mathrm{zz}(0,1$ and -1) Aromaticity results (in ppm) of the studied Chichibabin systems in the CS, OSS and T states. For the type-B rings (depicted in the scheme of Table S31).

\begin{tabular}{cccc|ccc|ccc}
\hline Structure & \multicolumn{1}{c}{ CICS(0) } & NICS(1) & NICS(-1) & NICS(0) & NICS(1) & NICS(-1) & NICS(0) & NICS(1) & NICS(-1) \\
h_4 & 3.7 & -14.5 & -14.5 & -6.3 & -22.0 & -22.0 & -7.2 & -22.6 & -22.6 \\
h_5 & -0.6 & -17.7 & -17.7 & -5.8 & -21.1 & -21.1 & -7.3 & -22.7 & -22.7 \\
\hline oh_4 & 4.3 & -14.0 & -14.0 & -4.1 & -20.3 & -20.3 & -5.7 & -21.5 & -21.5 \\
oh_5 & -0.1 & -17.3 & -17.3 & -5.7 & -21.5 & -21.5 & -5.9 & -21.7 & -21.7 \\
\hline nh_4 & 5.4 & -13.1 & -13.1 & -1.1 & -18.1 & -18.1 & -4.1 & -20.2 & -20.2 \\
nh_5 & 1.9 & -15.6 & -15.6 & -3.5 & -19.8 & -19.8 & -3.9 & -20.1 & -20.1 \\
\hline coh_4 & -0.8 & -17.9 & -17.9 & -6.4 & -22.0 & -22.0 & -7.1 & -22.5 & -22.5 \\
coh_5 & -3.7 & -20.0 & -20.0 & -6.9 & -22.4 & -22.4 & -7.1 & -22.5 & -22.5 \\
\hline cn_4 & 0.1 & -17.1 & -17.1 & -5.8 & -21.6 & -21.6 & -6.8 & -22.3 & -22.3 \\
cn_5 & -3.3 & -19.6 & -19.6 & -6.7 & -22.2 & -22.2 & -6.5 & -22.1 & -22.1 \\
\hline biph_4 & -5.5 & -21.3 & -21.3 & -8.2 & -23.3 & -23.3 & -8.3 & -23.4 & -23.4 \\
biph_5 & -7.1 & -22.5 & -22.5 & -8.4 & -23.4 & -23.4 & -8.4 & -23.4 & -23.4 \\
\hline ph_4 & -2.3 & -19.0 & -19.0 & -7.4 & -22.8 & -22.8 & -7.7 & -23.0 & -23.0 \\
ph_5 & -5.1 & -21.0 & -21.0 & -7.8 & -23.0 & -23.0 & -7.8 & -23.0 & -23.0 \\
\hline p_fph_4 & -2.4 & -19.0 & -19.0 & -7.4 & -22.8 & -22.8 & -7.7 & -22.9 & -22.9 \\
p_fph_5 & -5.2 & -21.1 & -21.1 & -7.7 & -23.0 & -23.0 & -7.8 & -23.0 & -23.0 \\
\hline p_noph_4 & -4.6 & -20.7 & -20.7 & -7.9 & -23.1 & -23.1 & -8.1 & -23.3 & -23.3 \\
p_noph_5 & -6.1 & -21.8 & -21.8 & -8.0 & -23.1 & -23.1 & -8.0 & -23.2 & -23.2 \\
\hline fph_4 & -0.9 & -17.9 & -17.9 & -7.0 & -22.4 & -22.4 & -7.5 & -22.8 & -22.8 \\
fph_5 & -4.1 & -20.2 & -20.2 & -7.4 & -22.7 & -22.7 & -7.5 & -22.8 & -22.8 \\
\hline noph_4 & -1.0 & -17.9 & -17.9 & -6.0 & -21.6 & -21.6 & -6.7 & -22.1 & -22.1 \\
noph_5 & -2.6 & -19.0 & -19.0 & -5.9 & -21.5 & -21.5 & -6.1 & -21.7 & -21.7 \\
\hline
\end{tabular}

Table S38. $\operatorname{NICS}_{Z Z}(0,1$ and -1) Aromaticity results (in ppm) of the studied Chichibabin systems in the $\mathrm{CS}$, OSS and T states. For the type-C rings (depicted in the scheme of Table S31).

\begin{tabular}{cccc:cccccc}
\hline Structure & NICS(0) & NICS(1) & NICS(-1) & NICS(0) & NICS(1) & NICS(-1) & NICS(0) & NICS(1) & NICS(-1) \\
h_3 & 9.2 & -10.3 & -10.3 & -1.7 & -18.6 & -18.6 & -7.1 & -22.6 & -22.6 \\
h_5 & -3.2 & -19.7 & -19.9 & -8.4 & -23.5 & -23.5 & -8.7 & -23.7 & -23.7 \\
\hline oh_3 & 9.8 & -9.9 & -9.9 & 4.4 & -14.0 & -14.0 & -5.3 & -21.3 & -21.3 \\
oh_5 & -2.4 & -19.0 & -19.0 & -7.6 & -22.9 & -22.9 & -7.9 & -23.1 & -23.1 \\
\hline nh_3 & 10.1 & -9.6 & -9.6 & 9.9 & -9.7 & -9.7 & -3.5 & -19.9 & -19.9 \\
nh_5 & -1.0 & -17.9 & -17.9 & -6.3 & -21.9 & -21.9 & -7.0 & -22.4 & -22.4 \\
\hline coh_3 & -1.5 & -18.2 & -18.2 & -5.7 & -32.1 & -32.2 & -6.4 & -22.0 & -22.0 \\
coh_5 & -5.6 & -21.4 & -21.4 & -8.4 & -23.4 & -23.4 & -8.5 & -23.6 & -23.6 \\
\hline cn_3 & 3.6 & -14.5 & -14.5 & -2.9 & -19.4 & -19.4 & -6.9 & -22.4 & -22.4 \\
cn_5 & -4.4 & -20.6 & -20.6 & -8.0 & -23.2 & -23.2 & -8.3 & -23.4 & -23.4 \\
\hline biph_3 & -1.7 & -18.5 & -18.5 & -7.5 & -22.8 & -22.8 & -8.1 & -23.2 & -23.2 \\
biph_5 & -8.0 & -23.1 & -23.1 & -9.0 & -23.9 & -23.9 & -9.0 & -23.9 & -23.9 \\
\hline ph_3 & 3.6 & -14.6 & -14.6 & -5.7 & -21.5 & -21.5 & -7.6 & -22.9 & -22.9 \\
ph_5 & -6.9 & -22.3 & -22.3 & -8.7 & -23.7 & -23.7 & -8.7 & -23.7 & -23.7 \\
\hline p_fph_3 & 3.5 & -14.7 & -14.7 & -5.5 & -21.4 & -21.4 & -7.6 & -22.9 & -22.9 \\
p_fph_5 & -6.9 & -22.3 & -22.3 & -8.7 & -23.7 & -23.7 & -8.7 & -23.7 & -23.7 \\
\hline p_noph_3 & 0.0 & -17.3 & -17.3 & -7.1 & -22.5 & -22.5 & -8.3 & -23.4 & -23.4 \\
p_noph_5 & -7.4 & -22.8 & -22.8 & -8.7 & -23.7 & -23.7 & -8.7 & -23.7 & -23.7 \\
\hline fph_3 & 5.1 & -13.3 & -13.3 & -4.6 & -20.6 & -20.6 & -7.3 & -22.6 & -22.6 \\
fph_5 & -6.0 & -21.7 & -21.7 & -8.5 & -23.5 & -23.5 & -8.6 & -23.6 & -23.6 \\
\hline noph_3 & 2.2 & -15.5 & -15.5 & -5.3 & -21.0 & -21.0 & -7.5 & -22.7 & -22.7 \\
noph_5 & -4.6 & -20.5 & -20.5 & -7.7 & -22.9 & -22.9 & -8.0 & -23.1 & -23.1 \\
\hline
\end{tabular}




\section{References}

[1] S. Grimme, J. Antony, S. Ehrlich, H. Krieg, A consistent and accurate ab initio parametrization of density functional dispersion correction (DFT-D) for the 94 elements H-Pu, J. Chem. Phys. 132 (2010) 154104.

[2] L. Noodleman, Valence bond description of antiferromagnetic coupling in transition metal dimers, J. Chem. Phys. 74 (1981) 5737-5743.

[3] M.J. Frisch, G.W. Trucks, H.B. Schlegel, G.E. Scuseria, M.A. Robb, J.R. Cheeseman, G. Scalmani, V. Barone, G.A. Petersson, H. Nakatsuji, X. Li, M. Caricato, A.V. Marenich, J. Bloino, B.G. Janesko, R. Gomperts, B. Mennucci, H.P. Hratchian, J.V. Ortiz, A.F. Izmaylov, J.L. Sonnenberg, Williams, F. Ding, F. Lipparini, F. Egidi, J. Goings, B. Peng, A. Petrone, T. Henderson, D. Ranasinghe, V.G. Zakrzewski, J. Gao, N. Rega, G. Zheng, W. Liang, M. Hada, M. Ehara, K. Toyota, R. Fukuda, J. Hasegawa, M. Ishida, T. Nakajima, Y. Honda, O. Kitao, H. Nakai, T. Vreven, K. Throssell, J.A. Montgomery Jr., J.E. Peralta, F. Ogliaro, M.J. Bearpark, J.J. Heyd, E.N. Brothers, K.N. Kudin, V.N. Staroverov, T.A. Keith, R. Kobayashi, J. Normand, K. Raghavachari, A.P. Rendell, J.C. Burant, S.S. Iyengar, J. Tomasi, M. Cossi, J.M. Millam, M. Klene, C. Adamo, R. Cammi, J.W. Ochterski, R.L. Martin, K. Morokuma, O. Farkas, J.B. Foresman, D.J. Fox, Gaussian 16 Rev. B.01, Wallingford, CT, 2016.

[4] B. Alexander Voigt, T. Steenbock, C. Herrmann, Structural diradical character, Journal of Computational Chemistry 40 (2019) 854-865.

[5] M. Güell, M. Solà, M. Swart, Spin-state splittings of iron(II) complexes with trispyrazolyl ligands, Polyhedron 29 (2010) 84-93.

[6] M. Swart, M. Güell, M. Solà, A multi-scale approach to spin crossover in Fe(ii) compounds, Physical Chemistry Chemical Physics 13 (2011) 10449-10456.

[7] Y. Zhang, A. Wu, X. Xu, Y. Yan, OPBE: A promising density functional for the calculation of nuclear shielding constants, Chemical Physics Letters 421 (2006) 383-388. 